\title{
Non-Negative Intensity for Structures with Inhomogeneous Damping
}

\author{
Daipei Liu \\ School of Mechanical and Manufacturing Engineering \\ The University of New South Wales \\ Sydney, New South Wales 2052, Australia \\ daipei.liu@unsw.edu.au \\ Steffen Marburg and Christian Geweth \\ Chair of Vibroacoustics of Vehicles and Machines \\ Department of Mechanical Engineering \\ Technische Universität München \\ Boltzmannstraße 15 \\ 85748 Garching, Germany \\ Nicole Kessissoglou \\ School of Mechanical and Manufacturing Engineering \\ The University of New South Wales \\ Sydney, New South Wales 2052, Australia
}

Received 4 February 2018

Accepted 5 August 2018

Published 7 September 2018

\begin{abstract}
In this work, numerical methods to identify the surface areas of a vibrating structure that radiate sound are implemented for cases of structures with inhomogeneous distributions of viscous Rayleigh damping. The intensity-based techniques correspond to acoustic intensity evaluated in terms of the acoustic pressure and particle velocity, non-negative intensity evaluated in terms of the acoustic impedance matrix obtained at the structural surface, and back-calculated non-negative intensity evaluated in terms of the acoustic impedance matrix obtained at a far-field receiver surface. Different configurations of inhomogeneous damping are applied to two elastic structures corresponding to a plate and a cylindrical shell. To examine the influence of inhomogeneous damping on sound radiation, the acoustic intensity on the structural surface, the acoustic intensity on several different far-field receiver surfaces, non-negative intensity and back-calculated non-negative intensity are numerically compared for different inhomogeneous damping cases.
\end{abstract}

Keywords: Acoustic intensity; non-negative intensity; inhomogeneous damping; radiated sound; boundary element method.

This is an Open Access article published by World Scientific Publishing Company. It is distributed under the terms of the Creative Commons Attribution 4.0 (CC-BY) License. Further distribution of this work is permitted, provided the original work is properly cited. 


\section{Liu et al.}

\section{Introduction}

To reduce exterior structure-borne sound, identification of the contribution to radiated sound from individual components of a vibrating structure is important. Acoustic intensity is usually used to analyze the contribution of a vibrating surface to the radiated sound power. Near-field acoustic holography (NAH) is an experimental technique to reconstruct the acoustic intensity on the surface of a radiating object from acoustic pressure measurements close to the vibrating surface! ${ }^{1}$ Using $\mathrm{NAH}$, the evanescent part of the wave spectrum is considered in the acoustic field reconstruction. Based on data processing of NAH in the wavenumber domain, Williams $\frac{23}{3}$ introduced the concept of supersonic acoustic intensity (SSI) to identify the wave components of the sound field propagating to the far field. After filtering out the subsonic wave components of the vibrating structure that only generate evanescent waves in the near field, the remaining supersonic components that radiate to the far field were identified. Based on the convolution theory, SSI for a planar geometry was obtained directly in the space domain, avoiding the need for Fourier transforms. 4 .5 Several numerical techniques have been developed to compute SSI of arbitrary geometries. 6 [ 8

The surface contribution method, first introduced by Marburg et al. ${ }^{[9}$ is an intensitybased technique to identify the contributions of local surface areas of a vibrating structure to far-field radiated sound. Using the acoustic impedance matrix obtained at the structural surface, the radiated sound power was computed as the sum of only positive sound power contributions of the vibrating structure, thus avoiding cancellation effects between positive and negative values of acoustic intensity from adjacent areas. Subsequently, the surface contribution method was renamed as non-negative intensity (NNI) by Williams.10 Results obtained using both NNI and SSI to identify the surface areas of arbitrarily-shaped vibrating structures that radiated sound have been recently compared using both a sound power ratio and the modal assurance criterion. ${ }^{11}$ Supersonic intensity was found to be difficult to implement at low frequencies due to the size of the spatial radiation filter. A cut-off coefficient associated with the acoustic wavenumber of the spatial radiation filter was used to reduce the aperture error of SSI. Similar distributions on the surface of a point excited plate were generated for NNI obtained numerically using the boundary element method and experimentally from NAH measurements. ${ }^{12}$ NNI has also been combined with the fast multipole boundary element method to consider large-scale fluid-structure interaction problems. 13

An extension to NNI is the back-calculated NNI, which is evaluated in terms of the acoustic impedance matrix obtained at a far-field receiver surface and as such, can identify surface contributions to sound power at a far-field surface that does not fully circumscribe the structure. ${ }^{1214}$ Back-calculated NNI evaluated at different far-field surfaces was compared to NNI for both rigid and elastic structures. NNI (always evaluated on the surface of the structure) and back-calculated NNI evaluated from a far-field surface that fully circumscribed the structure were shown to generate identical results. However, back-calculated NNI evaluated on different far-field surfaces that do not fully circumscribe the structure was found to generate markedly different results, highlighting the ability of back-calculated NNI to identify regions on a rigid or elastic structure that contribute to directional structure-borne sound. 
The use of NNI to identify local surface areas of a structure that radiate sound has only been implemented on structures with homogeneous damping distributions. For lightweight structures, a solution to increase dissipation rates of vibration energy to improve vibroacoustic behavior is the application of additional damping treatment such as constrained layer damping or embedded elastomer layers. When the spatial distribution of damping is inhomogeneous, it generates complex vibration modes which are no longer dominated by pure standing waves, but by the superposition of traveling and standing waves. ${ }^{15}$ Marburg evaluated the radiated sound power of an open cavity taking into account its complex modes. Sound radiation from vibrating structures with complex vibration modes was reported by Unruh et al! ${ }^{17[18}$ A finite element model of a simply-supported rectangular plate was developed to investigate the spatial distribution of acoustic intensity both on the plate surface and on a far-field surface. It was shown that traveling wave components of the complex modes can significantly influence the sound radiation. Unruh ${ }^{19}$ extended his investigation to consider complex mode radiation efficiencies for different inhomogeneous spatial damping configurations of the plate. Symmetric damping distributions were shown to have an almost negligible influence on sound radiation.

This paper examines, for the first time, surface contributions to radiated sound power from vibrating structures with inhomogeneous distributions of viscous Rayleigh damping. Several intensity-based numerical techniques corresponding to acoustic intensity, nonnegative intensity and back-calculated NNI are employed. A square plate and a cylindrical shell with symmetric and doubly symmetric configurations of inhomogeneous damping are examined. Results for the particle velocity distribution of a complex vibration mode for each of the inhomogeneous damping cases are compared to results for the case with homogeneous damping. The acoustic intensity on the structural surface as well as the acoustic intensity on several different far-field surfaces, corresponding to a spherical surface that fully circumscribes the structure and hemispherical surfaces at different orientations, are compared. Consideration of a partial receiver surface allows the surface contributions to radiated sound power integrated over a specific far-field region to be identified. Results obtained using NNI evaluated on the surface of the structure and back-calculated NNI on the far-field receiver surfaces for the homogeneous and inhomogeneous damping cases are compared to those for acoustic intensity. For the low frequencies considered in this work, NNI and back-calculated NNI show markedly different results to those obtained using acoustic intensity.

\section{Non-Negative Intensity}

Figure 1 shows a schematic diagram of structure-borne sound radiating from an arbitrarily shaped elastic structure to full or partial far-field receiver surface areas $\Gamma_{f f}$. Discretization of the acoustic domain using the collocation boundary element method leads to the following linear relation between the acoustic pressure $\mathbf{p}_{\Gamma}$ and the particle velocity $\mathbf{v}_{\Gamma}$ on the structural surface $\Gamma_{\Gamma} 20$

$$
\mathbf{H}_{\Gamma} \mathbf{p}_{\Gamma}=\mathbf{G}_{\Gamma} \mathbf{v}_{\Gamma}
$$




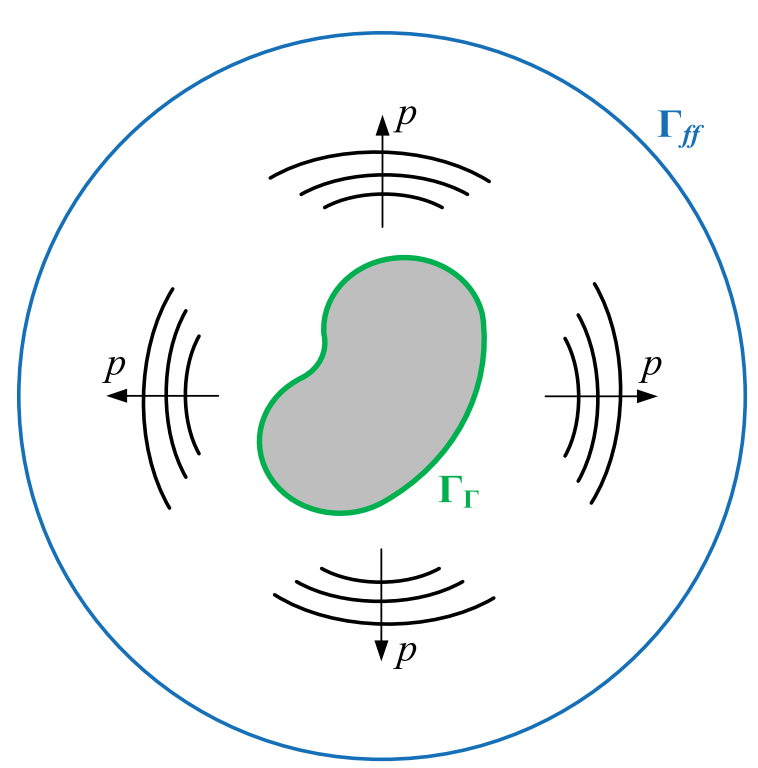

(a)

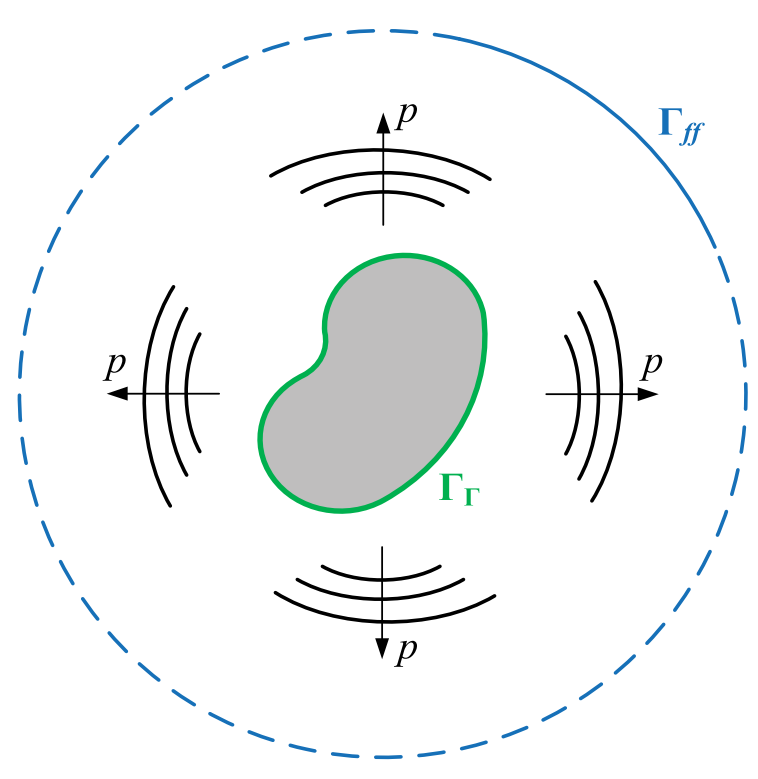

(b)

Fig. 1. Acoustic radiation by an elastic structure showing: (a) a full far-field receiver surface area and (b) a partial far-field receiver surface area.

where the subscript ()$_{\Gamma}$ denotes a quantity evaluated on the structural surface mesh with $N$ collocation points. $\mathbf{G}_{\Gamma}$ and $\mathbf{H}_{\Gamma}$ are boundary element matrices on the structural surface. The vector of nodal values for active acoustic intensity $\mathbf{I}_{\Gamma}$ on the structural surface $\Gamma_{\Gamma}$ is defined as

$$
\mathbf{I}_{\Gamma}=\frac{1}{2} \operatorname{Re}\left\{p_{\Gamma} \mathbf{v}_{\Gamma}^{*}\right\}
$$

where ()$^{*}$ denotes the complex conjugate. The radiated sound power is calculated via a surface integral of the acoustic intensity over the structural surface $\Gamma_{\Gamma}$ as

$$
P_{\Gamma}=\int_{\Gamma_{\Gamma}} \mathbf{I}_{\Gamma} \cdot \mathbf{n}_{\Gamma} \mathrm{d} \Gamma_{\Gamma}
$$

where $\mathbf{n}_{\Gamma}$ is the normal direction on $\Gamma_{\Gamma}$.

The acoustic intensity field calculated using Eq. (2.2) consists of both positive and negative components and as such does not provide a clear indication of the local surface contributions of energy to the radiated sound power. The radiated sound power can be alternatively expressed in terms of the NNI $I_{\Gamma}^{\mathrm{NNI}}(\mathbf{x})$ as 9111

$$
P_{\Gamma}^{\mathrm{NNI}}=\int_{\Gamma_{\Gamma}} I_{\Gamma}^{\mathrm{NNI}}(\mathbf{x}) \mathrm{d} \Gamma_{\Gamma}=\int_{\Gamma_{\Gamma}} \frac{1}{2} \beta_{\Gamma}(\mathbf{x}) \beta_{\Gamma}^{*}(\mathbf{x}) \mathrm{d} \Gamma_{\Gamma},
$$

where $\mathbf{x}$ is the field point. $\beta_{\Gamma}(\mathbf{x})$ is a quantity without physical significance and is computed as follows ${ }^{9}$

$$
\beta_{\Gamma}(\mathbf{x})=\phi_{\Gamma}^{\top}(\mathbf{x}) \mathbf{\Psi}_{\Gamma} \sqrt{\boldsymbol{\Lambda}_{\Gamma}} \mathbf{\Psi}_{\Gamma}^{\top} \boldsymbol{\Theta}_{\Gamma} \mathbf{v}_{\Gamma} .
$$


$\Theta_{\Gamma}$ is a diagonal matrix of the element areas on the structural surface and is defined as $20-22$

$$
\Theta_{\Gamma}=\int_{\Gamma_{\Gamma}} \phi_{\Gamma} \phi_{\Gamma}^{\top} \mathrm{d} \Gamma_{\Gamma}
$$

where $\phi_{\Gamma}$ is a column matrix containing the interpolation function and ()$^{\top}$ denotes the matrix transpose. $\boldsymbol{\Psi}_{\Gamma}$ and $\boldsymbol{\Lambda}_{\Gamma}$ are the set of eigenvectors and corresponding diagonal matrix of eigenvalues found by solving the following algebraic eigenvalue problem $\frac{9] 12[14}{1.14}$

$$
\mathbf{Z}_{\Gamma, R} \psi_{\Gamma}=\lambda_{\Gamma} \Theta_{\Gamma} \psi_{\Gamma}
$$

where $\lambda_{\Gamma}$ are the individual eigenvalues. $\mathbf{Z}_{\Gamma, R}$ is the real part of the acoustic impedance matrix obtained on the structural surface $\mathbf{Z}_{\Gamma}$, where $\mathbf{Z}_{\Gamma}$ is defined as 9

$$
\mathbf{Z}_{\Gamma}=\mathbf{G}_{\Gamma}^{\top} \mathbf{H}_{\Gamma}^{-\top} \boldsymbol{\Theta}_{\Gamma}
$$

\section{Back-Calculated Non-Negative Intensity}

The acoustic pressure $p_{f f}$ and the fluid particle velocity $\mathbf{v}_{f f}$ at the nodes of a full or partial far-field surface mesh with $M$ collocation points can be expressed in terms of the particle velocity on the structural surface $\mathbf{v}_{\Gamma}$ as follows 20

$$
\begin{aligned}
& \mathbf{p}_{f f}=\mathbf{G}_{\Gamma}-\mathbf{H} \mathbf{p}_{\Gamma}=\left(\mathbf{G}-\mathbf{H H}_{\Gamma}^{-1} \mathbf{G}_{\Gamma}\right) \mathbf{v}_{\Gamma}=-\mathbf{B}_{\mathrm{pv}} \mathbf{v}_{\Gamma}, \\
& \mathbf{v}_{f f}=\mathbf{G}^{\prime} \mathbf{v}_{\Gamma}-\mathbf{H}^{\prime} \mathbf{p}_{\Gamma}=\left(\mathbf{G}^{\prime}-\mathbf{H}^{\prime} \mathbf{H}_{\Gamma}^{-1} \mathbf{G}_{\Gamma}\right) \mathbf{v}_{\Gamma}=-\mathbf{B}_{\mathrm{vv}} \mathbf{v}_{\Gamma},
\end{aligned}
$$

where the subscript ()$_{f f}$ denotes a quantity evaluated on the far-field surface mesh. $\mathbf{G}$ and $\mathbf{H}$ are boundary element coefficient matrices in the acoustic domain and $\mathbf{G}^{\prime}, \mathbf{H}^{\prime}$ are the normal derivatives of matrices $\mathbf{G}$ and $\mathbf{H}$, respectively. $\mathbf{B}_{\mathrm{pv}}, \mathbf{B}_{\mathrm{vv}}, \mathbf{G}^{\prime}$ and $\mathbf{H}^{\prime}$ are all $M \times N$ matrices.

The vector of nodal values for the active acoustic intensity on the far-field receiver surface $\Gamma_{f f}$ is defined as $12 \mid 23$

$$
\mathbf{I}_{f f}=\frac{1}{2} \operatorname{Re}\left\{p_{f f} \mathbf{v}_{f f}^{*}\right\} .
$$

The radiated sound power based on acoustic intensity is defined as $\frac{9121}{10}$

$$
P_{f f}=\int_{\Gamma_{f f}} \mathbf{I}_{f f} \cdot \mathbf{n}_{f f} \mathrm{~d} \Gamma_{f f},
$$

where $\mathbf{n}_{f f}$ is the normal direction on $\Gamma_{f f}$.

Back-calculated NNI is an intensity-based technique that can identify surface contributions to sound power at a far-field receiver surface that does not fully circumscribe the structure. To obtain back-calculated NNI denoted by $I_{f f}^{\mathrm{NNI}}(\mathbf{x})$, the radiated sound power in Eq. (3.4) can be expressed as 9

$$
P_{f f}^{\mathrm{NNI}}=\int_{\Gamma_{\Gamma}} I_{f f}^{\mathrm{NNI}}(\mathbf{x}) \mathrm{d} \Gamma_{\Gamma}(\mathbf{x})=\int_{\Gamma_{\Gamma}} \frac{1}{2} \beta_{f f}(\mathbf{x}) \beta_{f f}^{*}(\mathbf{x}) \mathrm{d} \Gamma_{\Gamma}(\mathbf{x}) .
$$


$\beta_{f f}(\mathbf{x})$ is an $N \times N$ matrix and is computed as follows 14

$$
\beta_{f f}(\mathbf{x})=\phi_{\Gamma}^{\top}(\mathbf{x}) \boldsymbol{\Psi}_{f f} \sqrt{\boldsymbol{\Lambda}_{f f}} \boldsymbol{\Psi}_{f f}^{\top} \boldsymbol{\Theta}_{\Gamma} \mathbf{v}_{\Gamma},
$$

where $\boldsymbol{\Psi}_{f f}$ and $\boldsymbol{\Lambda}_{f f}$ are the set of eigenvectors and corresponding diagonal matrix of eigenvalues found by solving the eigenvalue problem of the acoustic impedance matrix obtained on the far-field surface $\mathbf{Z}_{f f}$, which in turn is defined as

$$
\mathbf{Z}_{f f}=\mathbf{B}_{\mathrm{pv}}^{\top} \Theta_{f f} \mathbf{B}_{\mathrm{vv}}^{*} .
$$

$\boldsymbol{\Theta}_{f f}$ is a diagonal matrix of the element areas on a far-field surface. The far-field receiver can be a surface that fully circumscribes the structure as shown in Fig. 1(a), or a partial surface that does not fully circumscribe the structure as shown in Fig. 1(b). As such, the radiated sound power $P_{f f}^{N N I}$ in Eq. (3.5) is the total radiated sound power or partial radiated sound power for the full or partial surface areas, respectively. Consideration of a partial receiver surface allows the surface regions radiating to a specific far-field receiver surface to be identified.

\section{Viscous Damping}

Finite element models for a square plate and a cylindrical shell were used to obtain the complex vibration modes of a structure with different configurations of inhomogeneous damping. In the FEM analysis, the damping matrix $\mathbf{D}$ was modeled using viscous Rayleigh damping and expressed as a linear combination of the mass and stiffness matrices as follows $\$ 24$

$$
\mathbf{D}=\alpha_{k} \mathbf{K}+\alpha_{m} \mathbf{M}
$$

where $\alpha_{k}$ and $\alpha_{m}$ are Rayleigh coefficients. $\mathbf{K}$ and $\mathbf{M}$ are respectively the stiffness and mass matrices. To obtain inhomogeneous damping, different regions of the structural surface with different Rayleigh damping ratios $\zeta_{i}$ are defined. The frequency-dependent Rayleigh damping ratio $\zeta_{i}$ is given by

$$
\zeta_{i}(\omega)=\frac{\alpha_{k, i}}{2 \omega}+\frac{\alpha_{m, i} \omega}{2}, \quad i=1,2 .
$$

A low Rayleigh damping ratio of $\zeta_{1}=0.01$ was applied to a structure with homogeneous damping. An artificially high Rayleigh damping ratio of $\zeta_{2}=0.3$ was then applied to various regions of the structural surface for the cases with inhomogeneous damping, whereby the significantly higher damping ratio was implemented in order to observe sufficient complexity of the resulting complex modes. The damping solver (DAMP) in ANSYS was used to obtain the damping matrix and complex eigenvectors for the plate and cylindrical shell with different distributions of inhomogeneous damping.

\section{Numerical Examples}

Two numerical examples are investigated corresponding to a square plate and a cylindrical shell with hemispherical end closures in air. Density $1.3 \mathrm{~kg} / \mathrm{m}^{3}$ and speed of sound $340 \mathrm{~m} / \mathrm{s}$ were assumed for air. The boundary element code AKUSTA ${ }^{26 \mid 27}$ was used to generate all 
boundary element matrices and boundary element coefficient vectors. Equations used for all other quantities in the acoustic domain were implemented using MATLAB.

\subsection{Square plate}

A $1 \mathrm{~m} \times 1 \mathrm{~m} \times 10 \mathrm{~mm}$ plate with clamped boundary conditions was discretized with 2432 shell 281 elements. Material properties of aluminium were used for the plate corresponding to Young's modulus of $70 \mathrm{GPa}$, density of $2710 \mathrm{~kg} / \mathrm{m}^{3}$ and Poisson's ratio of 0.35 . Five different configurations of inhomogeneous damping were applied to the plate corresponding to cases 2-6 in Fig. 2 whereby the higher damping regions are marked in blue. Case 1 represents the reference case of the plate with homogeneous damping. Cases 2 and 3 represent inhomogeneous damping with symmetric damping distributions. Case 4 represents inhomogeneous damping with bilateral symmetric damping distribution about one axis. Cases 5 and 6 represent inhomogeneous damping with symmetric damping distributions about one diagonal. The spatial distribution of the inhomogeneous damping is the same on the top and bottom surfaces of the plate.

Figure 3 presents the real part, imaginary part and normalized magnitude of the particle velocity on the plate surface for the $(3 \times 3)$ mode of the plate. The nomenclature of the $(3 \times 3)$ mode is based on the number of nodal lines. Case 1 corresponding to the plate with homogeneous damping shows that the particle velocity only has an imaginary component which is dominated by standing waves, resulting in a real normal mode. For the cases with inhomogeneous damping (cases 2-6), the particle velocity has both real and imaginary components which include the superposition of both standing and traveling waves! $15 / 18 / 19$ Traveling waves can be observed in the imaginary part in case 3 and the real part in cases 4 and 5, showing that the traveling waves propagate towards the higher damping area, as previously reported $\frac{19}{19}$ Further, since the particle velocity distributions occur at both the top and bottom surfaces of the plate, acoustic waves at a given frequency traveling from

Case 1

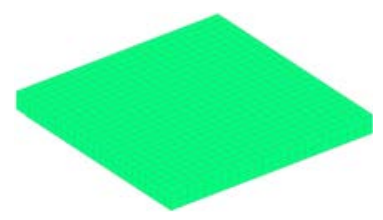

Case 4

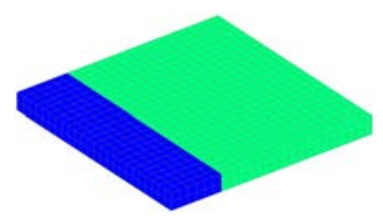

Case 2

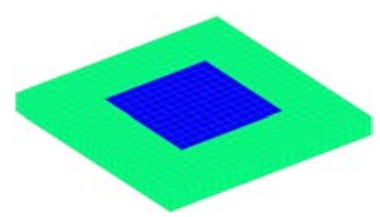

Case 5

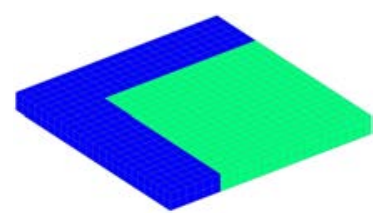

Case 3

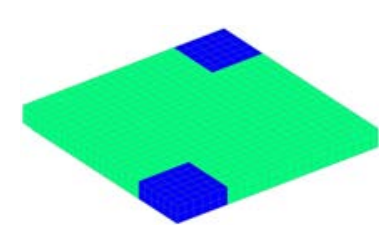

Case 6

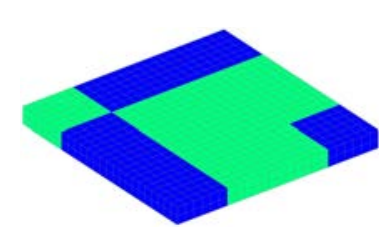

Fig. 2. A homogeneous plate (case 1) and five different configurations of inhomogeneous damping applied to the plate (cases 2-6). 

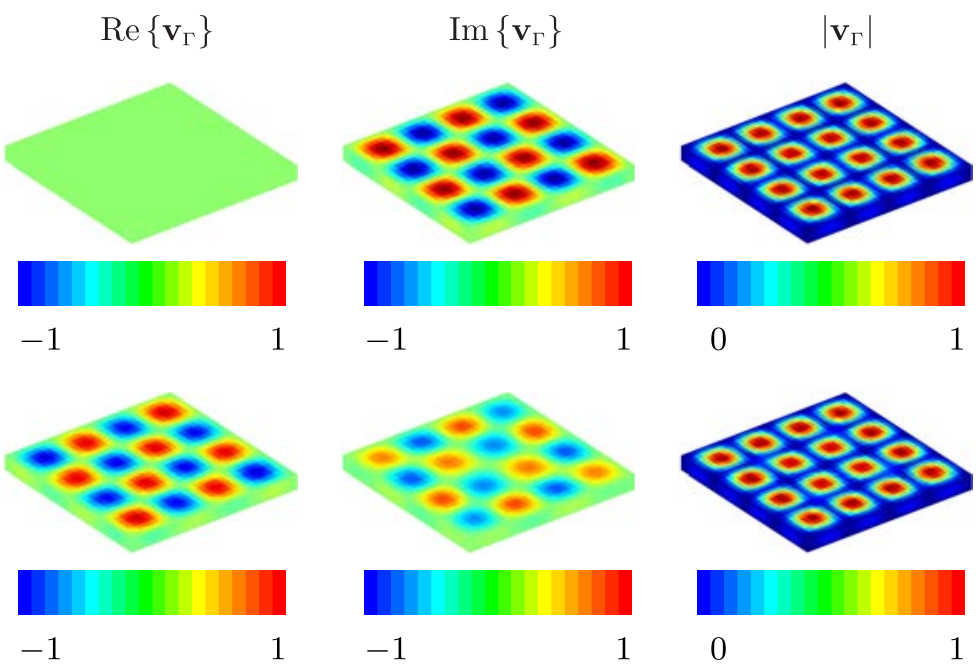

Case 2
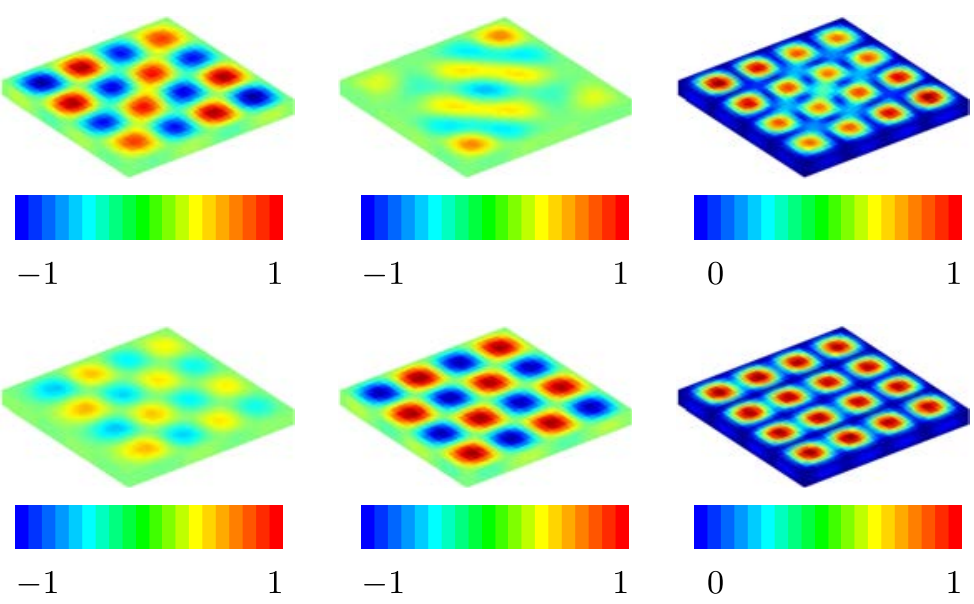

Case 4
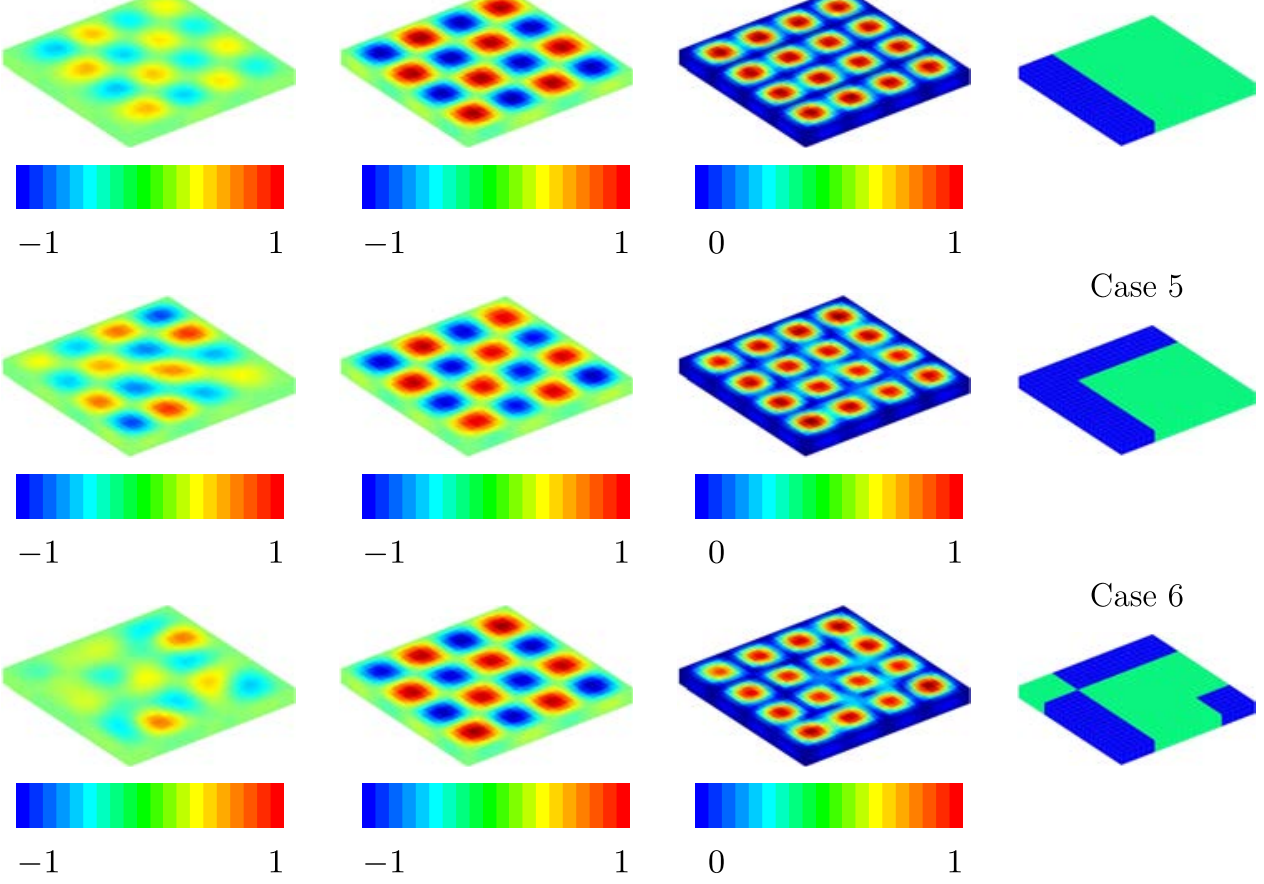

Fig. 3. The real part, imaginary part and normalized magnitude of the particle velocity for the $(3 \times 3)$ mode of the plate with different damping configurations (velocity in $\mathrm{mm} / \mathrm{s}$ ). 
the top and bottom surfaces cancel each other at the middle layer of the plate. Hence, the thickness of the plate has a negligible influence on the intensity results. The results presented here assume that normal and complex vibration modes can occur at any possible frequency below the coincidence frequency, at which the acoustic wavelength is equal to the wavelength of the flexural waves in the plate. It was previously reported that results for NNI approach those for acoustic intensity at high frequencies due to the decrease in the acoustic wavelength and corresponding increase in the acoustic radiation efficiency! ${ }^{911}$ To better highlight differences between acoustic intensity and NNI for the inhomogeneous damping cases, a low frequency of $100 \mathrm{~Hz}$ was selected.

For the particle velocity distributions in Fig. 3, results for acoustic intensity on the plate surface $\mathbf{I}_{\Gamma}$, acoustic intensity on several different far-field receiver surfaces $\mathbf{I}_{f f}$, NNI evaluated on the plate surface $\mathbf{I}_{\Gamma}^{\mathrm{NNI}}$, and back-calculated NNI evaluated from the different far-field surfaces $\mathbf{I}_{f f}^{\mathrm{NNI}}$, are now examined. Figure 4 presents the active acoustic intensity on the
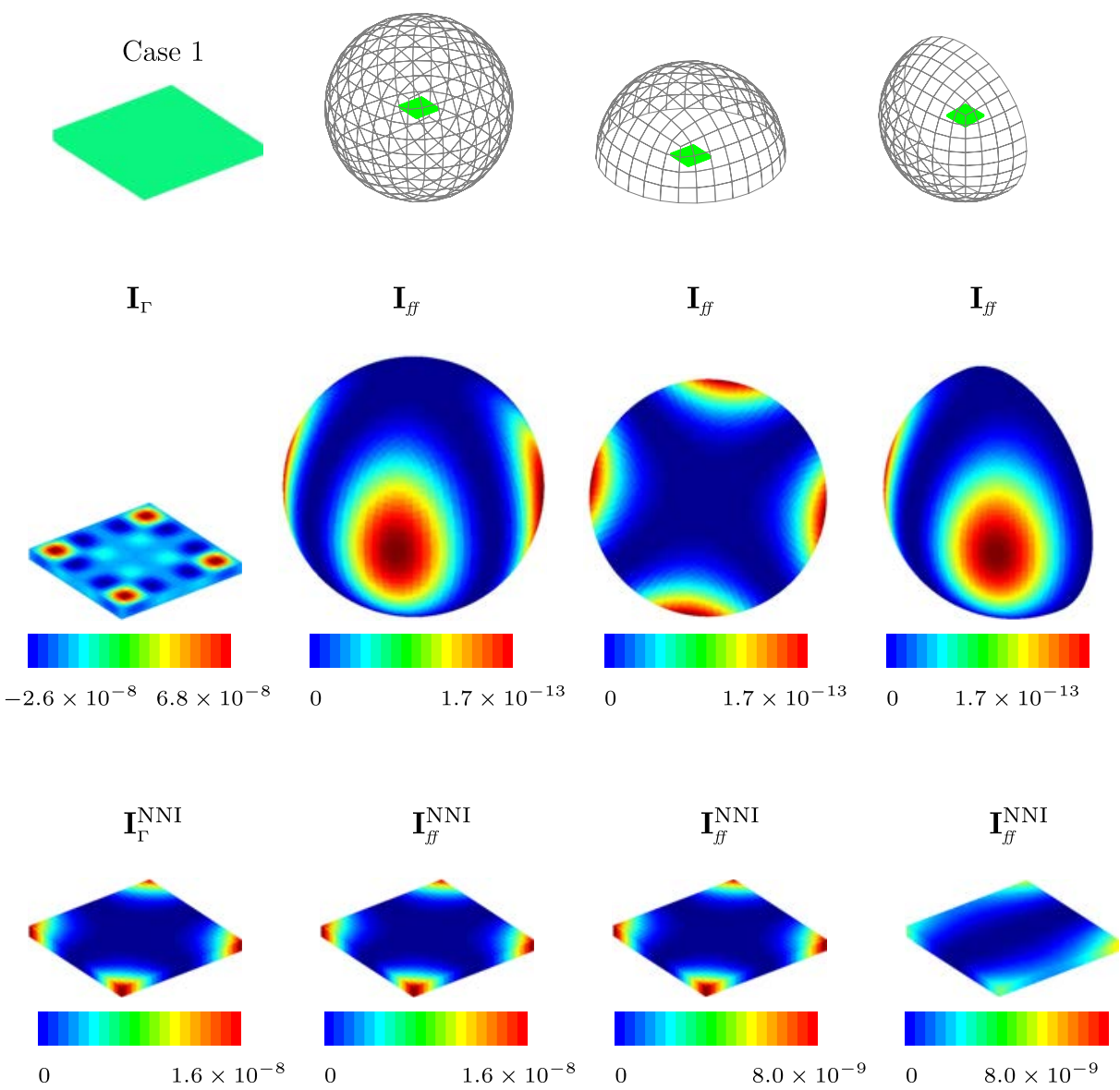

Fig. 4. Acoustic intensity at the structural surface $\mathbf{I}_{\Gamma}$, acoustic intensity at the far-field receiver surfaces $\mathbf{I}_{f f}$, NNI $\mathbf{I}_{\Gamma}^{\mathrm{NNI}}$, and back-calculated NNI $\mathbf{I}_{f f}^{\mathrm{NNI}}$, for the $(3 \times 3)$ real normal mode of the plate at $100 \mathrm{~Hz}$ (intensity in $\left.\mathrm{W} / \mathrm{m}^{2}\right)$. 


\section{Liu et al.}

surface of the plate as well as the active acoustic intensity on three far-field receiver surfaces corresponding to a spherical surface that fully circumscribes the plate, a top hemispherical surface and a left hemispherical surface, for the $(3 \times 3)$ mode of the plate with homogeneous damping. The diameter of the far-field spherical and hemispherical surfaces is $200 \mathrm{~m}$, with the plate at the center of the spherical surfaces. To more clearly show the distribution pattern, results for the acoustic intensity on the top hemispherical surface are presented from the top view. For the plate with homogeneous damping, the acoustic intensity on the far-field spherical surface shows maximum values occurring at four distinct equatorial locations. Similar results for the acoustic intensity can also be observed on the top and left hemispherical surfaces. The minimum values of the acoustic intensity are positive and several orders of magnitude smaller than the maximum values; as such the minimum values are marked as zero. Further, the maximum values of the acoustic intensity on the far-field surfaces are identical.

Figure 4 also presents results for NNI as well as back-calculated NNI evaluated from the far-field receiver surfaces, highlighting local surface contributions to the radiated sound power. NNI and back-calculated NNI evaluated from a far-field spherical surface that fully circumscribes the plate exhibit identical radiation patterns of the same maximum value. The plate corner radiation is due to cancelation of acoustic energy between adjacent areas. This result can be observed for all odd-odd order bending modes of the plate at low frequencies,

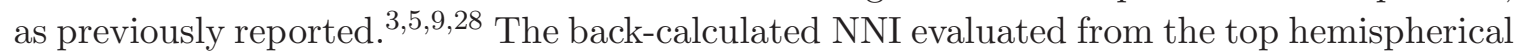
surface shows the same distribution pattern compared to the back-calculated NNI evaluated from the full spherical surface although its maximum value is approximately half. The backcalculated NNI evaluated from the left hemispherical surface highlights plate edges with higher intensity coinciding with the maximum acoustic intensity that occurs at the far-field hemispherical surface.

Similar to Fig. 4. Figs. 50 present the active acoustic intensity on the surface of the plate $\mathbf{I}_{\Gamma}$, active acoustic intensity on the three far-field receiver surfaces $\mathbf{I}_{f f}$, NNI evaluated on the plate surface $\mathbf{I}_{\Gamma}^{\mathrm{NNI}}$, and back-calculated NNI evaluated from the far-field surfaces $\mathbf{I}_{f f}^{\mathrm{NNI}}$, for the $(3 \times 3)$ mode of the plate with different damping configurations. The active acoustic intensity on the structural surface $\mathbf{I}_{\Gamma}$ exhibits both positive and negative values, where the negative values highlight the higher damping regions for each case. Regions with lower damping act as acoustic sources with positive acoustic intensity, whereas higher damping regions dissipate bending waves and become energy sinks, as previously reported.17 For case 2 (Fig. 5), the acoustic intensity on the far-field surface shows almost the same distribution to the result for the plate with homogeneous damping (Fig. 4). NNI and backcalculated NNI also show similar results to those of the real normal mode for the case with homogeneous damping. As previously reported by Unruh, $\frac{19}{19}$ symmetric damping distributions have an almost negligible influence on sound radiation. For cases 3-6 (Figs. 6 9]), it can be observed that the directivity of the far-field acoustic intensity loses its quadrupole characteristic. Interestingly, the directivity of far-field acoustic intensity is consistent with the distribution of the higher damping regions, showing that the higher damping regions 

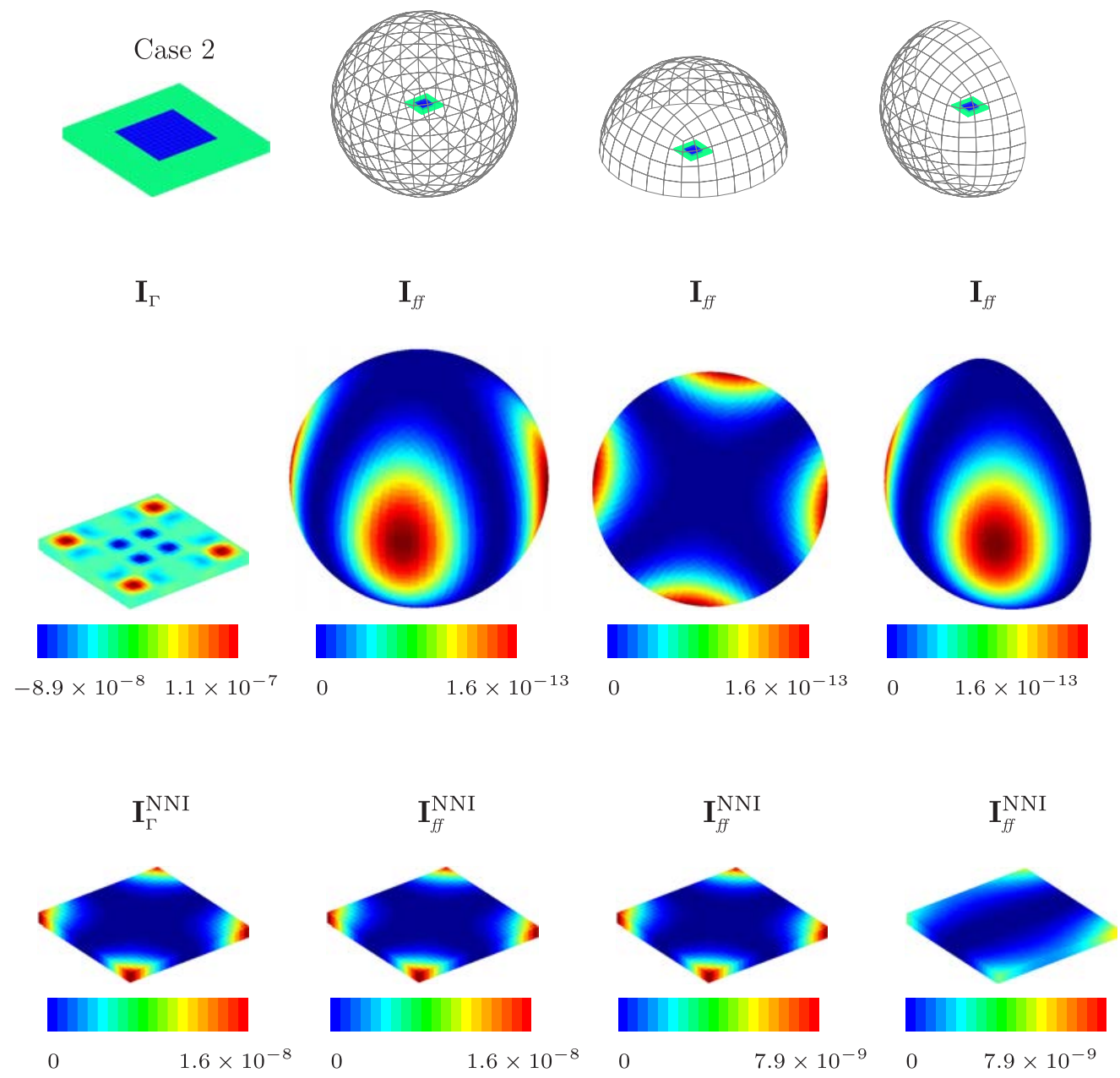

Fig. 5. Acoustic intensity at the structural surface $\mathbf{I}_{\Gamma}$, acoustic intensity at the far-field receiver surfaces $\mathbf{I}_{f f}$, NNI $\mathbf{I}_{\Gamma}^{\mathrm{NNI}}$, and back-calculated NNI $\mathbf{I}_{f f}^{\mathrm{NNI}}$, for the $(3 \times 3)$ complex mode of the plate at $100 \mathrm{~Hz}$ (intensity in $\left.\mathrm{W} / \mathrm{m}^{2}\right)$.

appear to contribute more energy to the far field. This result is in contrast to the acoustic intensity on the surface of the plate, for which the higher damping regions become intensity sinks. This is attributed to the fact that sound radiated from acoustic source and sink regions partially cancel each other and only a fraction of the acoustic energy close to the plate surface reaches the far field. Case 6 (Fig. 9) shows that the directivity of far-field acoustic intensity shifts toward the opposite direction compared to the result for case 5 (Fig. 8), due to the shift in higher damping to the opposite diagonal. For each damping configuration, NNI and back-calculated NNI evaluated from the spherical receiver surface are identical. In case 3 (Fig. 6), NNI and back-calculated NNI indicate that two diagonal corners with higher intensity close to the maximum acoustic intensity contribute maximum 

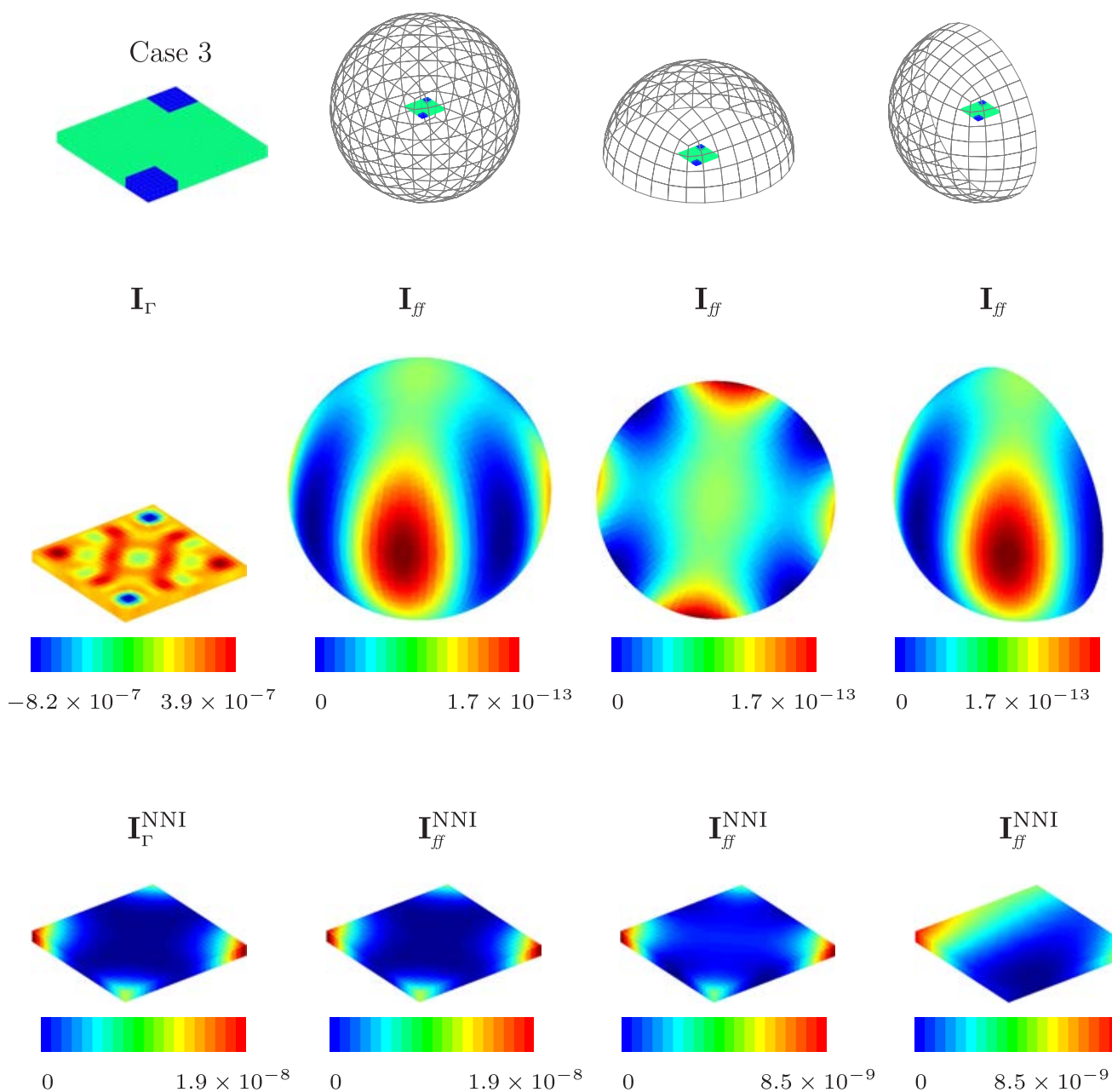

Fig. 6. Acoustic intensity at the structural surface $\mathbf{I}_{\Gamma}$, acoustic intensity at the far-field receiver surfaces $\mathbf{I}_{f f}$, $\mathrm{NNI} \mathbf{I}_{\Gamma}^{\mathrm{NNI}}$, and back-calculated NNI $\mathbf{I}_{f f}^{\mathrm{NNI}}$, for the $(3 \times 3)$ complex mode of the plate at $100 \mathrm{~Hz}$ (intensity in $\left.\mathrm{W} / \mathrm{m}^{2}\right)$.

energy, which is in contrast to the regions of greatest intensity at the far-field receiver surfaces. Inspection of the imaginary part of the particle velocity for case 3 in Fig. 3 reveals that traveling waves propagate towards the diagonal corners with higher localized damping. As a result, the opposite diagonal corners of the plate exhibit higher magnitude of the particle velocity, yielding both greater positive-only acoustic intensity and NNI at these corners. In cases 4-6, results for NNI identify surface contributions at the plate corners corresponding to regions with higher values of both positive and negative acoustic intensity. As such, NNI avoids the cancellation effects between the positive and negative acoustic intensity from adjacent areas on the plate. Results for back-calculated NNI for cases 3-6 are also notably different to the results for acoustic intensity on the far-field receiver surfaces, depending on shape and orientation of the far-field surface. 

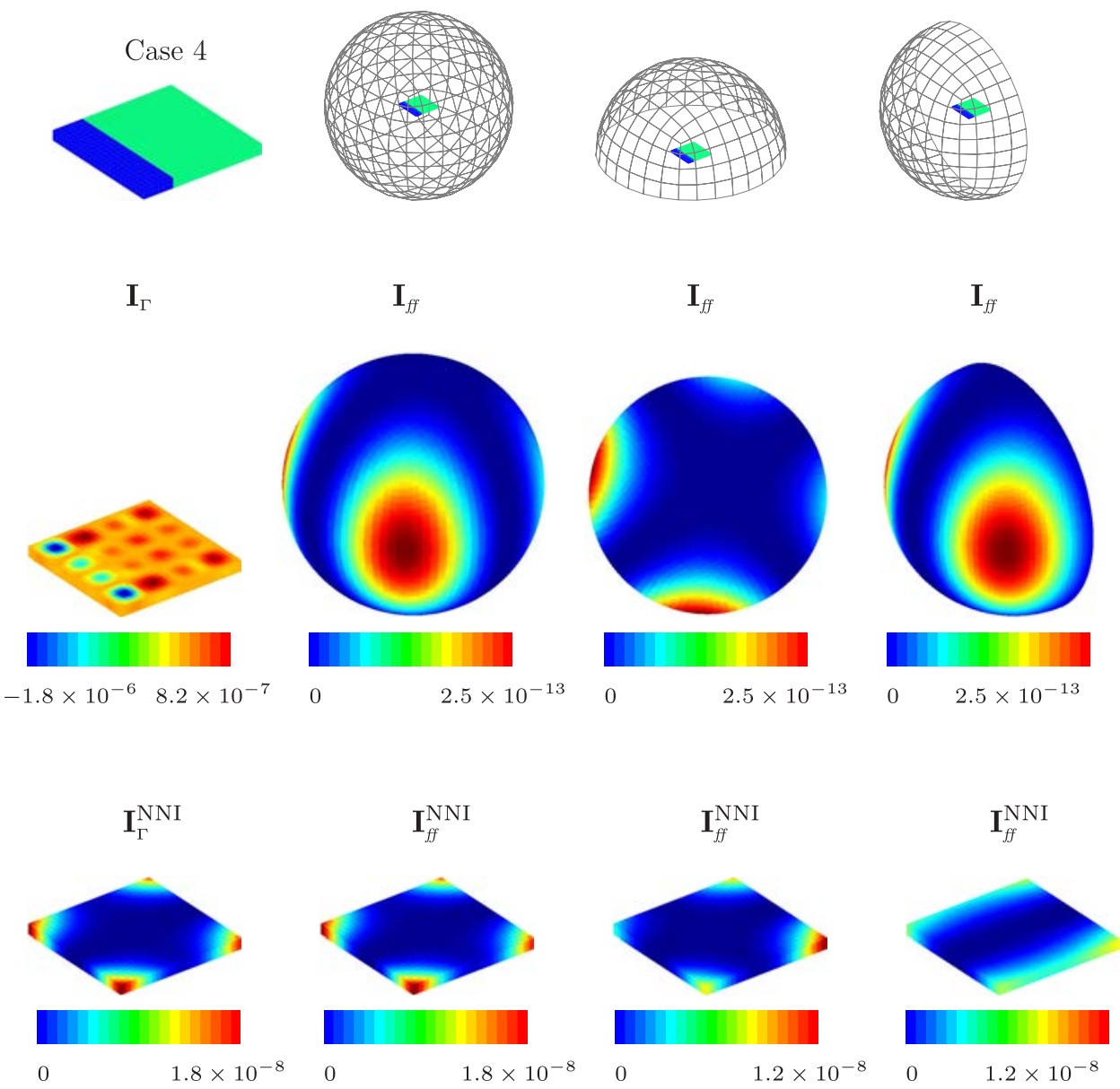

Fig. 7. Acoustic intensity at the structural surface $\mathbf{I}_{\Gamma}$, acoustic intensity at the far-field receiver surfaces $\mathbf{I}_{f f}$, $\mathrm{NNI} \mathbf{I}_{\Gamma}^{\mathrm{NNI}}$, and back-calculated NNI $\mathbf{I}_{f f}^{\mathrm{NNI}}$, for the $(3 \times 3)$ complex mode of the plate at $100 \mathrm{~Hz}$ (intensity in $\left.\mathrm{W} / \mathrm{m}^{2}\right)$.

\subsection{Cylindrical shell}

A cylindrical shell closed with hemispherical end caps with free boundary conditions was discretized with 2304 shell 281 elements. The material properties are the same as those for the aluminium plate. The cylindrical shell has radius of $0.5 \mathrm{~m}$, length of $4 \mathrm{~m}$ and thickness of $3 \mathrm{~mm}$. Five different configurations of inhomogeneous damping were also applied to the cylindrical shell corresponding to cases $2-6$ as shown in Fig. 10, whereby the higher damping regions are marked in blue. Case 1 represents the reference case of the cylindrical shell with homogeneous damping. Cases 2 and 3 represent inhomogeneous damping with symmetric damping distribution about both the longitudinal and circumferential axes. Case 4 represents inhomogeneous damping with symmetric damping distribution about the circumferential axis, and similarly, cases 5 and 6 represent inhomogeneous damping with symmetric damping distributions about the longitudinal axis. 

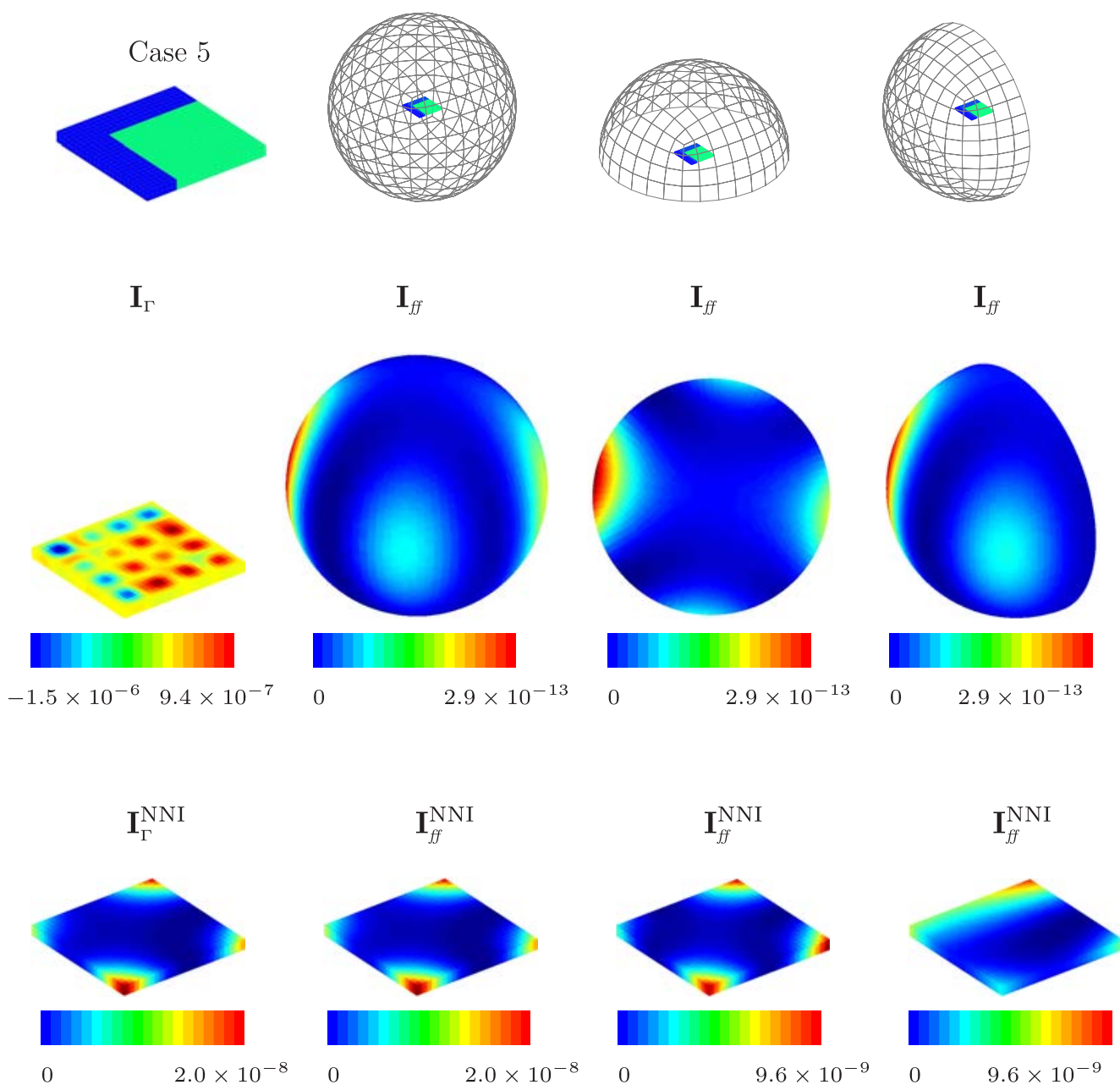

Fig. 8. Acoustic intensity at the structural surface $\mathbf{I}_{\Gamma}$, acoustic intensity at the far-field receiver surfaces $\mathbf{I}_{f f}$, NNI $\mathbf{I}_{\Gamma}^{\mathrm{NNI}}$, and back-calculated NNI $\mathbf{I}_{f f}^{\mathrm{NNI}}$, for the $(3 \times 3)$ complex mode of the plate at $100 \mathrm{~Hz}$ (intensity in $\left.\mathrm{W} / \mathrm{m}^{2}\right)$.

Figure 11 presents the real part, imaginary part and normalized magnitude of the particle velocity on the structural surface at the second bending mode of the cylindrical shell with different damping configurations. The cylindrical shell with homogeneous damping (case 1) shows that the particle velocity only has an imaginary component which is dominated by standing waves, corresponding to the second bending mode of the cylindrical shell. For the cases with inhomogeneous damping (cases 2-6), the particle velocity has both real and imaginary components. The imaginary components show the distribution pattern of the second bending mode, while the real components exhibit more complex patterns.

A low frequency of $20 \mathrm{~Hz}$ was selected to evaluate the intensity results of the cylindrical shell. Three different far-field receiver surfaces corresponding to a spherical surface 

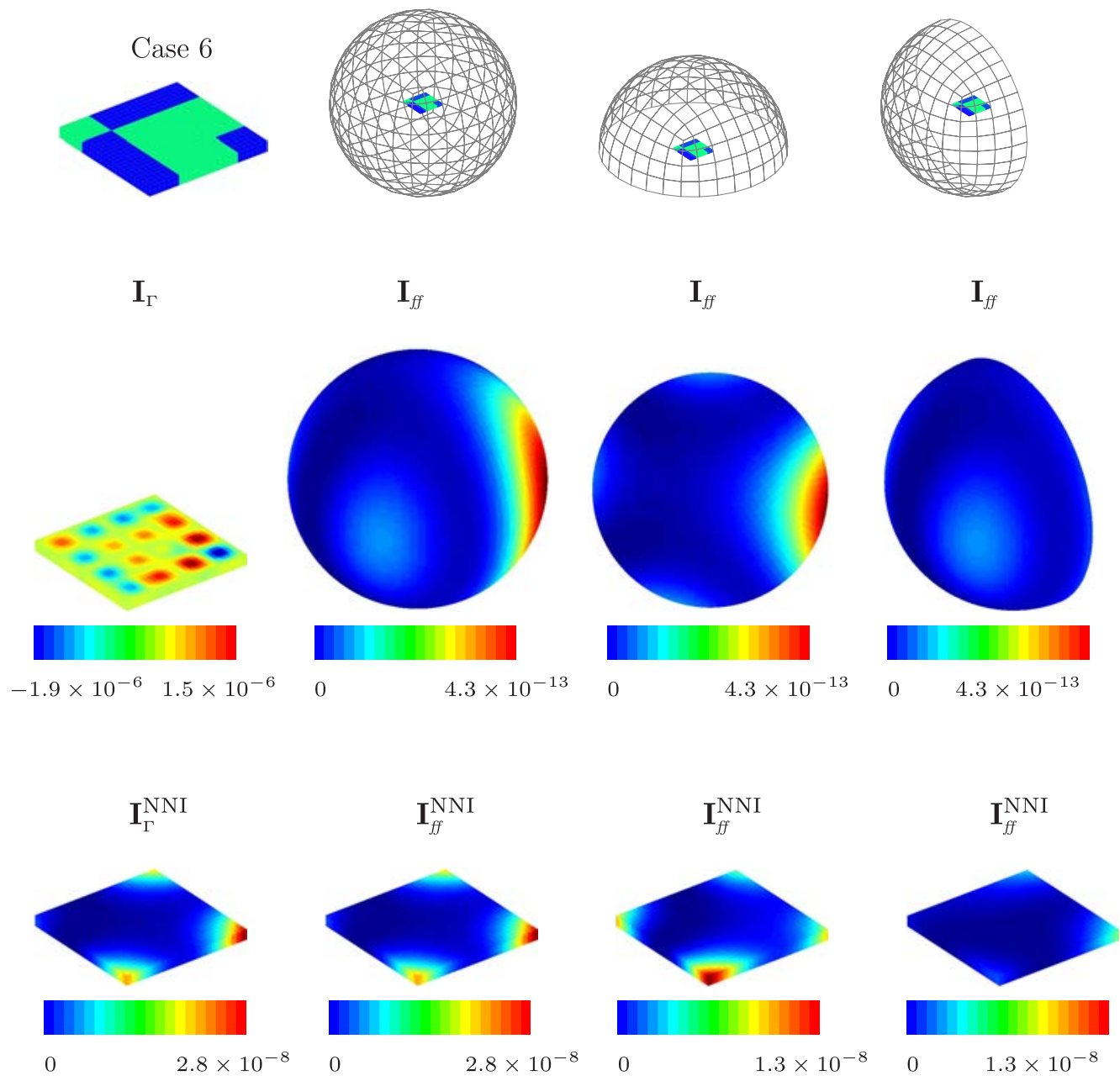

Fig. 9. Acoustic intensity at the structural surface $\mathbf{I}_{\Gamma}$, acoustic intensity at the far-field receiver surfaces $\mathbf{I}_{f f}$, NNI $\mathbf{I}_{\Gamma}^{\mathrm{NNI}}$, and back-calculated NNI $\mathbf{I}_{f f}^{\mathrm{NNI}}$, for the $(3 \times 3)$ complex mode of the plate at $100 \mathrm{~Hz}$ (intensity in $\left.\mathrm{W} / \mathrm{m}^{2}\right)$.

of $200 \mathrm{~m}$ diameter, a right hemispherical surface and a top hemispherical surface of the same size diameter were used to evaluate the acoustic intensity and the back-calculated NNI. Figure 12 presents the active acoustic intensity on the surface of the cylindrical shell $\mathbf{I}_{\Gamma}$, the active acoustic intensity on the three far-field surfaces $\mathbf{I}_{f f}$, NNI on the cylindrical shell surface $\mathbf{I}_{\Gamma}^{\mathrm{NNI}}$, and back-calculated NNI evaluated from the three far-field surfaces $\mathbf{I}_{f f}^{\mathrm{NNI}}$, for case 1 corresponding to the cylindrical shell with homogeneous damping. The acoustic intensity on the far-field spherical receiver surface shows maximum values occurring at four distinct circumferential locations. Similar to the results of the plate, NNI and back-calculated NNI evaluated from a far-field spherical surface that fully circumscribes the cylindrical shell exhibit identical radiation patterns, showing that only areas around the junction of the cylindrical and hemispherical shells at both ends are radiating sound. 


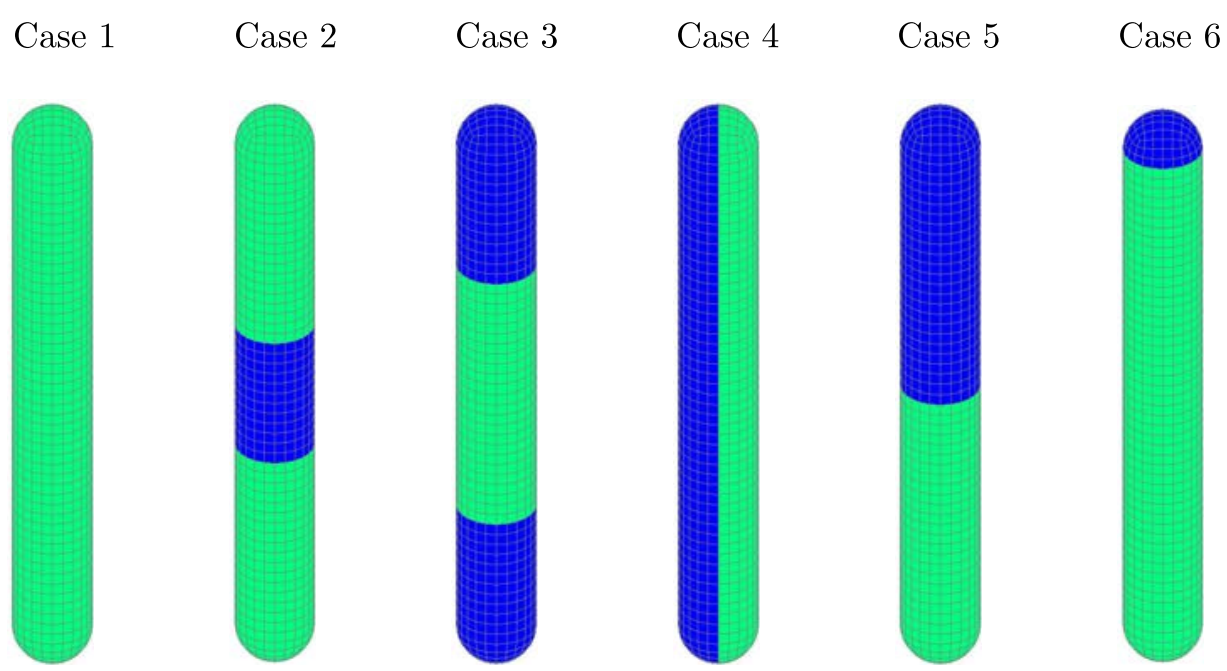

Fig. 10. A homogeneous cylindrical shell and four different configurations of inhomogeneous damping applied to the cylindrical shell.

This radiation pattern is referred to as an edge mode, as previously reported,,$[6]$ and can be observed for all even order bending modes of the cylindrical shell at low frequencies. The back-calculated NNI evaluated from the right hemispherical surface shows the same distribution pattern compared to the back-calculated NNI evaluated from the full spherical surface although its maximum value is approximately half due to the symmetric loading on the cylindrical shell. The back-calculated NNI evaluated from the top hemispherical surface highlights both sides of the cylindrical shell with higher intensity.

Figures 13 17 present the active acoustic intensity on the surface of the cylindrical shell $\mathbf{I}_{\Gamma}$, active acoustic intensity on the three far-field receiver surfaces $\mathbf{I}_{f f}$, NNI on the cylindrical shell surface $\mathbf{I}_{\Gamma}^{\mathrm{NNI}}$, and back-calculated NNI evaluated from three far-field surfaces $\mathbf{I}_{f f}^{\mathrm{NNI}}$, for the cylindrical shell with different damping configurations. The negative values of the acoustic intensity on the cylindrical shell surface highlight that the higher damping regions acts as energy sinks for each case. In case 2 (Fig. 13) and case 3 (Fig. 14), the active acoustic intensity on the far-field surfaces show almost the same distribution to the results for the cylindrical shell with homogeneous damping (Fig. 12). This is attributed to the complex modes arising from the doubly symmetric damping distribution not significantly affecting the sound radiation. For cases $4-6$, greater acoustic intensity on the far-field receiver surfaces is observed to consistently occur with the higher damping regions on the cylindrical shell. It is also observed that the quadrupole directivity characteristic associated with the lower damping regions is lessened. For each damping configuration, NNI and back-calculated NNI evaluated from the spherical receiver surface are identical. Results for NNI and backcalculated NNI for cases 2 and 3 are similar to those of the case with homogeneous damping (case 1) due to the doubly symmetric damping distributions. For cases 4-6, NNI and backcalculated NNI show that the cylinder edge radiation still dominates to a greater or lesser degree depending on the damping distribution. 


$$
\operatorname{Re}\left\{\mathbf{v}_{\Gamma}\right\} \quad \operatorname{Im}\left\{\mathbf{v}_{\Gamma}\right\} \quad\left|\mathbf{v}_{\Gamma}\right| \quad \operatorname{Re}\left\{\mathbf{v}_{\Gamma}\right\} \quad \operatorname{Im}\left\{\mathbf{v}_{\Gamma}\right\} \quad\left|\mathbf{v}_{\Gamma}\right|
$$

Case 1

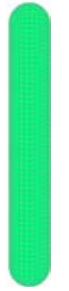

Case 3

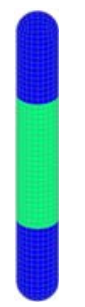

Case 5
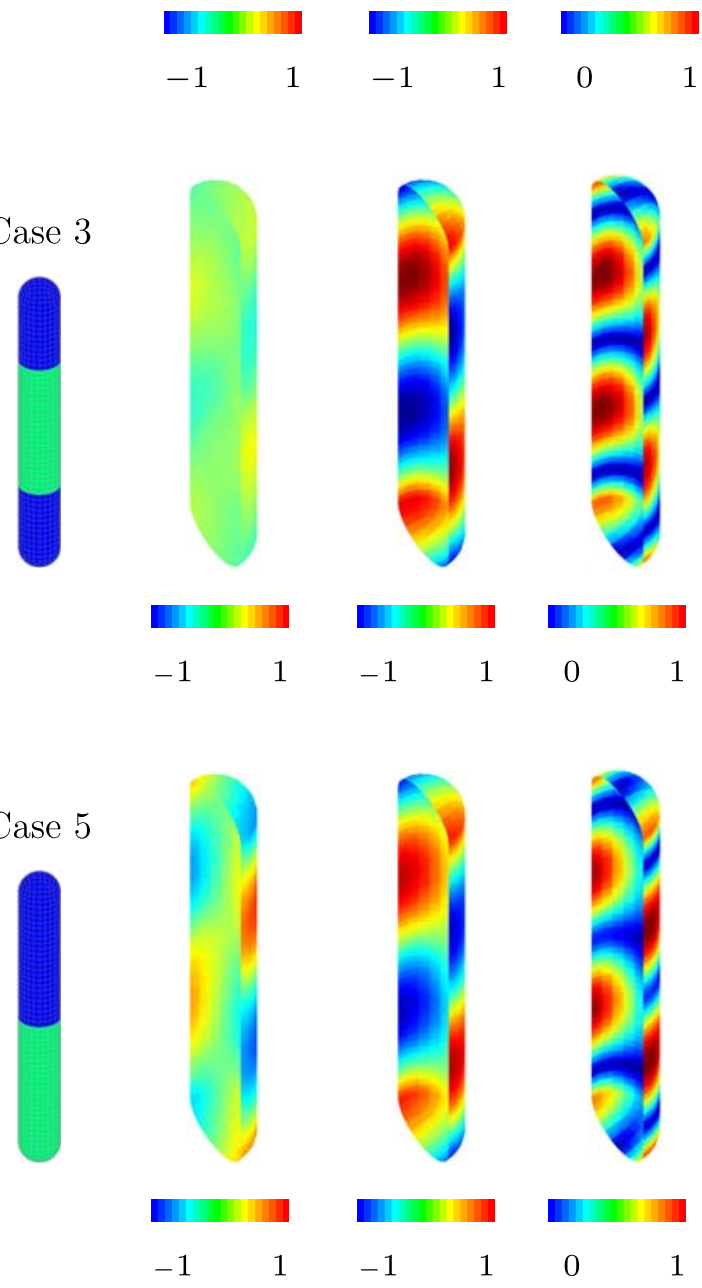
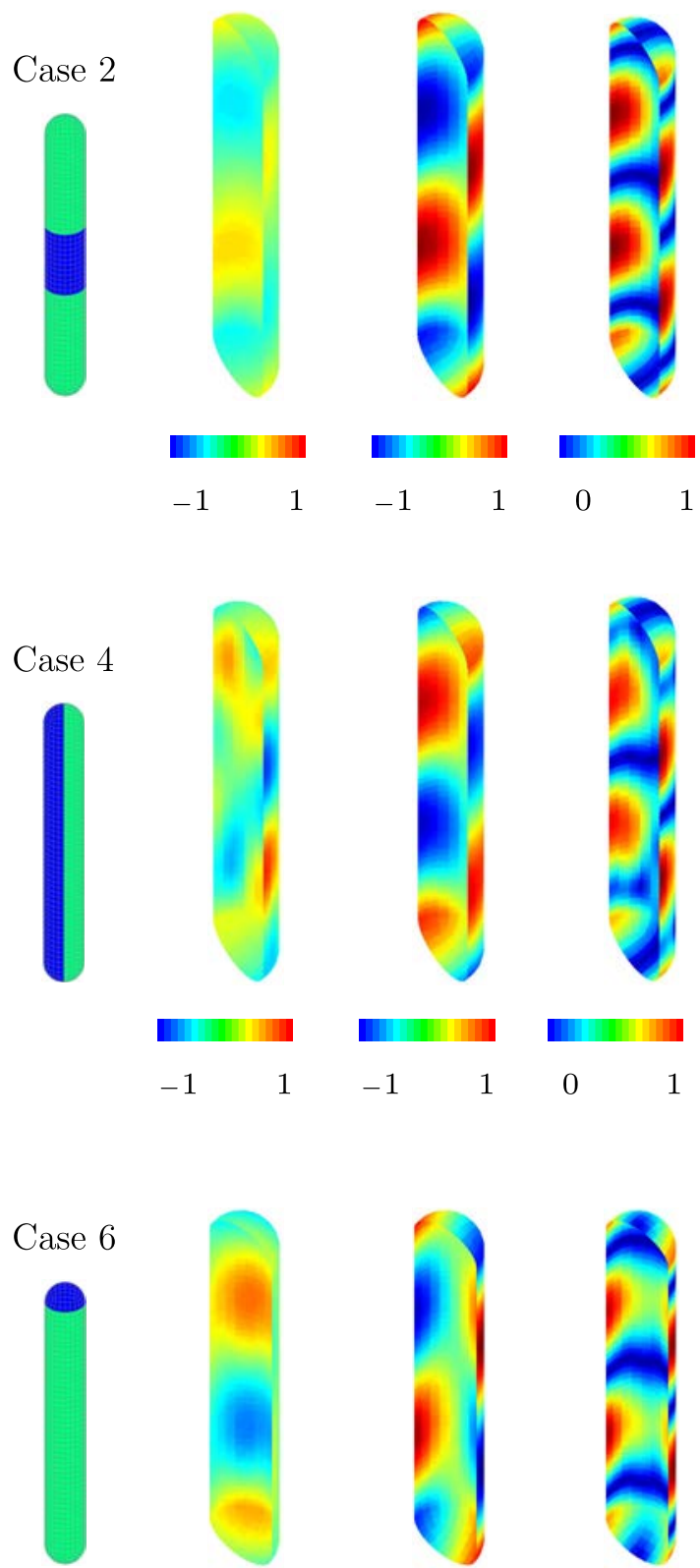

Fig. 11. The real part, imaginary part and normalized magnitude of the particle velocity at the second bending mode of the cylindrical shell with different damping configurations (velocity in $\mathrm{mm} / \mathrm{s}$ ). 
D. Liu et al.

Case 1
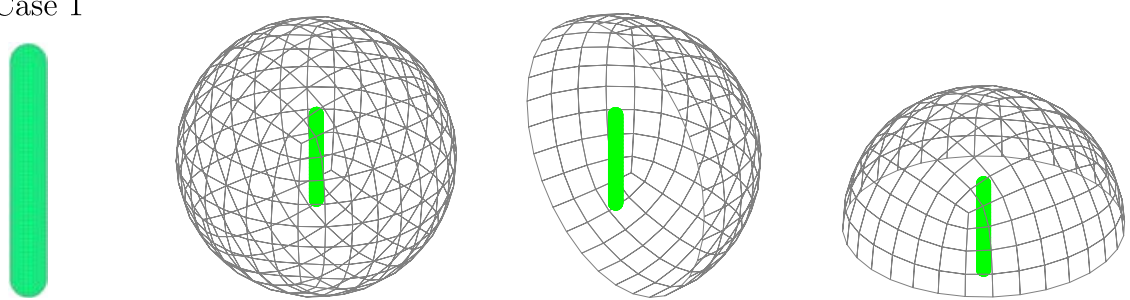

$\mathbf{I}_{\Gamma}$

$\mathbf{I}_{f f}$

$\mathbf{I}_{f f}$

$\mathbf{I}_{f f}$
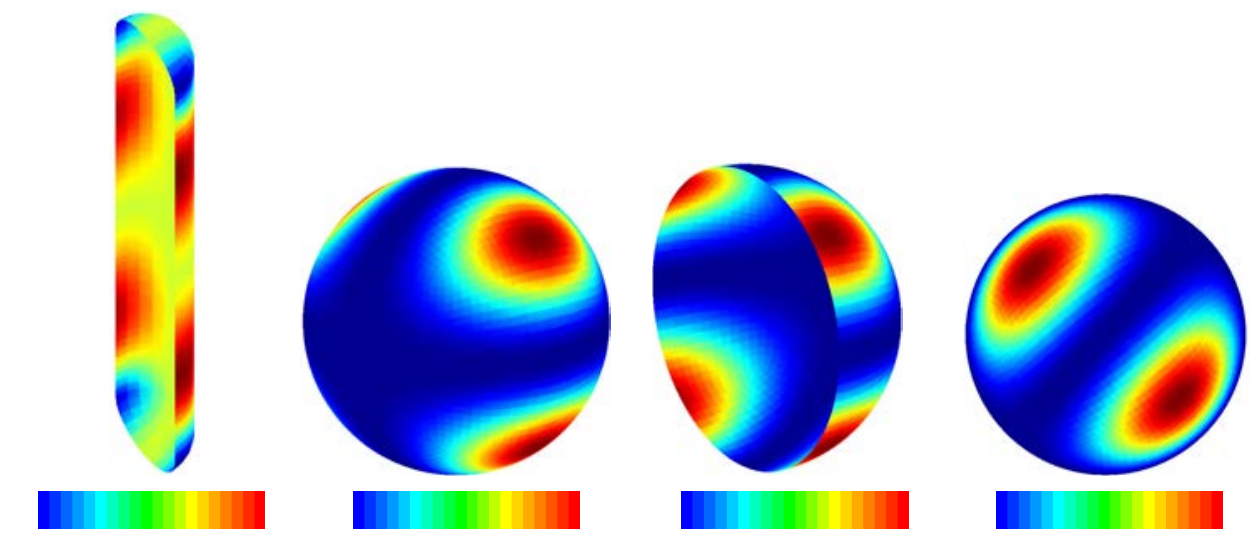

$-4.1 \times 10^{-7} 3.0 \times 10^{-7}$

0

$5.4 \times 10^{-15}$

0

$5.4 \times 10^{-15}$

$0 \quad 5.4 \times 10^{-15}$

$\mathbf{I}_{\Gamma}^{\mathrm{NNI}}$

$\mathbf{I}_{f f}^{\mathrm{NNI}}$

$\mathbf{I}_{f f}^{\mathrm{NNI}}$

$\mathbf{I}_{f f}^{\mathrm{NNI}}$
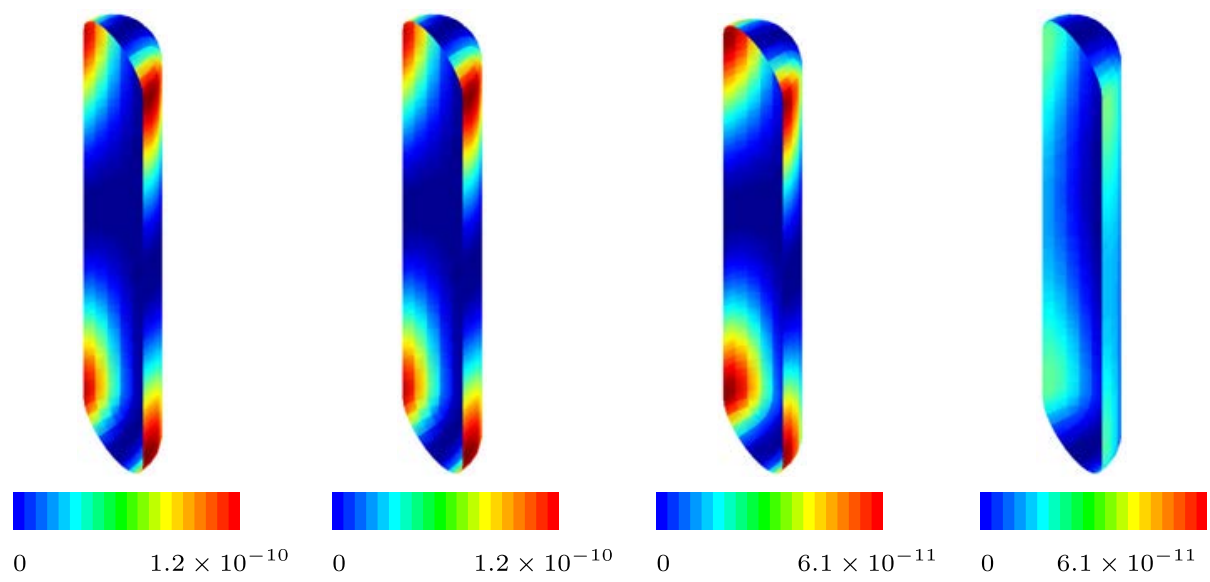

Fig. 12. Acoustic intensity at the structural surface $\mathbf{I}_{\Gamma}$, acoustic intensity at the far-field receiver surfaces $\mathbf{I}_{f f}$, NNI $\mathbf{I}_{\Gamma}^{\mathrm{NNI}}$, and back-calculated NNI $\mathbf{I}_{f f}^{\mathrm{NNI}}$, for the second bending mode of the cylindrical shell at $20 \mathrm{~Hz}$ (intensity in $\mathrm{W} / \mathrm{m}^{2}$ ). 
Case 2
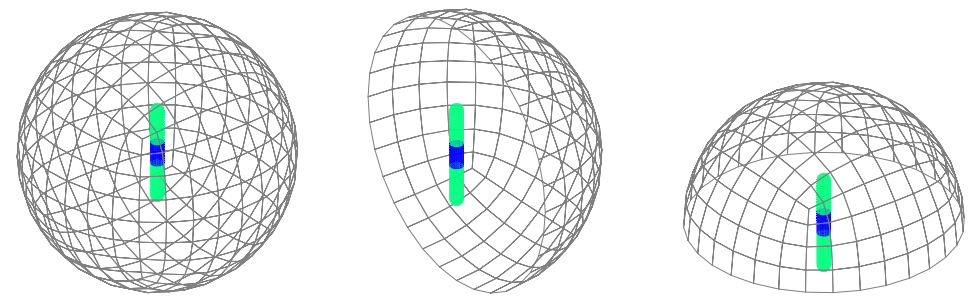

$\mathbf{I}_{\Gamma}$

$\mathbf{I}_{f f}$

$\mathbf{I}_{f f}$

$\mathbf{I}_{f f}$
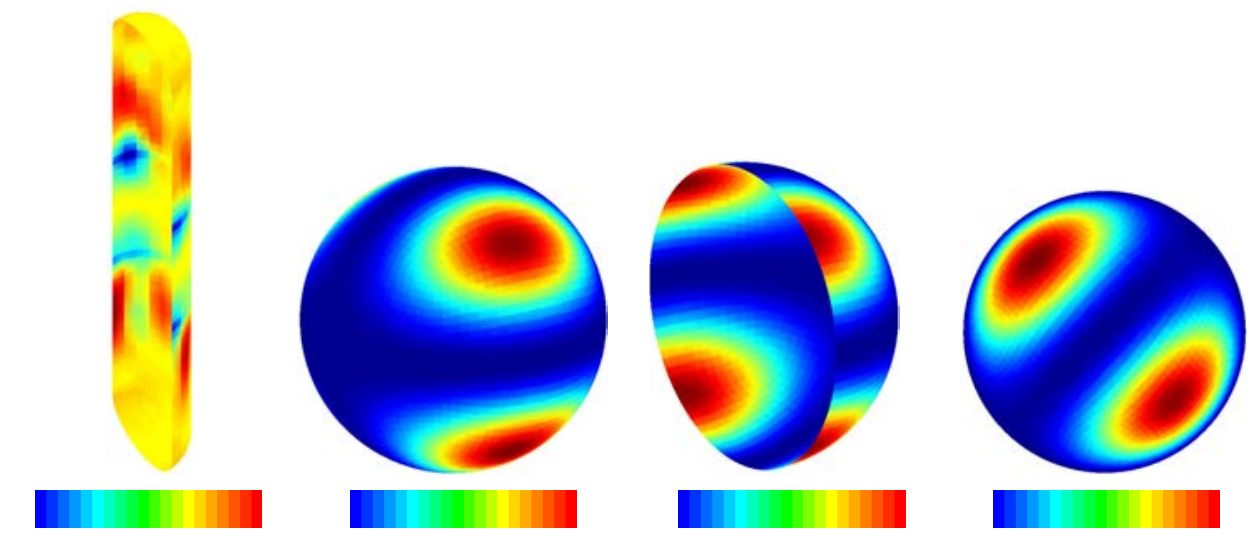

$-2.4 \times 10^{-7} 1.5 \times 10^{-7}$

0

$5.6 \times 10^{-15}$

0

$5.6 \times 10^{-15}$

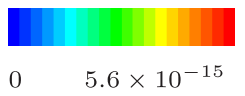

$\mathbf{I}_{\Gamma}^{\mathrm{NNI}}$

$\mathbf{I}_{f f}^{\mathrm{NNI}}$

$\mathbf{I}_{f f}^{\mathrm{NNI}}$

$\mathbf{I}_{f f}^{\mathrm{NNI}}$
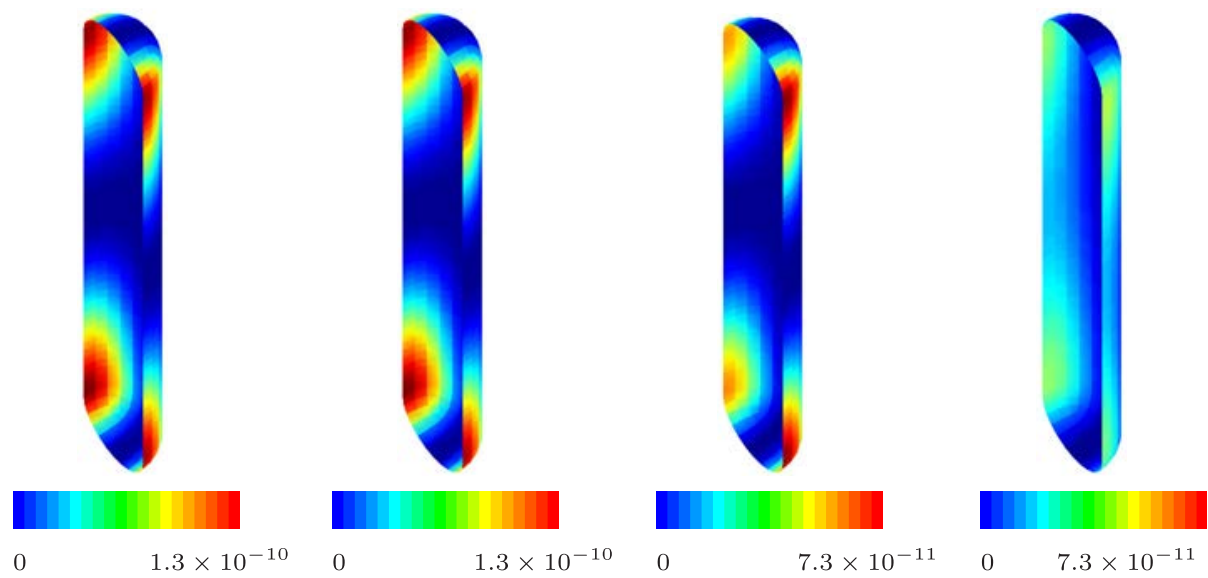

Fig. 13. Acoustic intensity at the structural surface $\mathbf{I}_{\Gamma}$, acoustic intensity at the far-field receiver surfaces $\mathbf{I}_{f f}$, NNI $\mathbf{I}_{\Gamma}^{\mathrm{NNI}}$, and back-calculated NNI $\mathbf{I}_{f f}^{\mathrm{NNI}}$, for the second bending complex mode of the cylindrical shell at $20 \mathrm{~Hz}$ (intensity in $\mathrm{W} / \mathrm{m}^{2}$ ). 
D. Liu et al.

Case 3
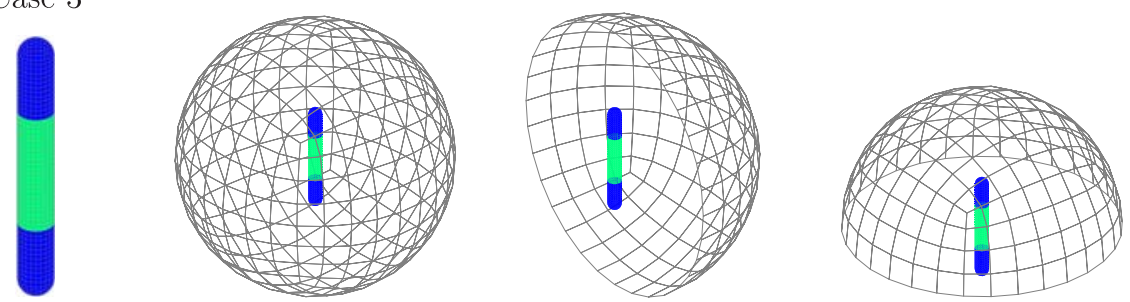

$\mathbf{I}_{\Gamma}$

$\mathbf{I}_{f f}$

$\mathbf{I}_{f f}$

$\mathbf{I}_{f f}$
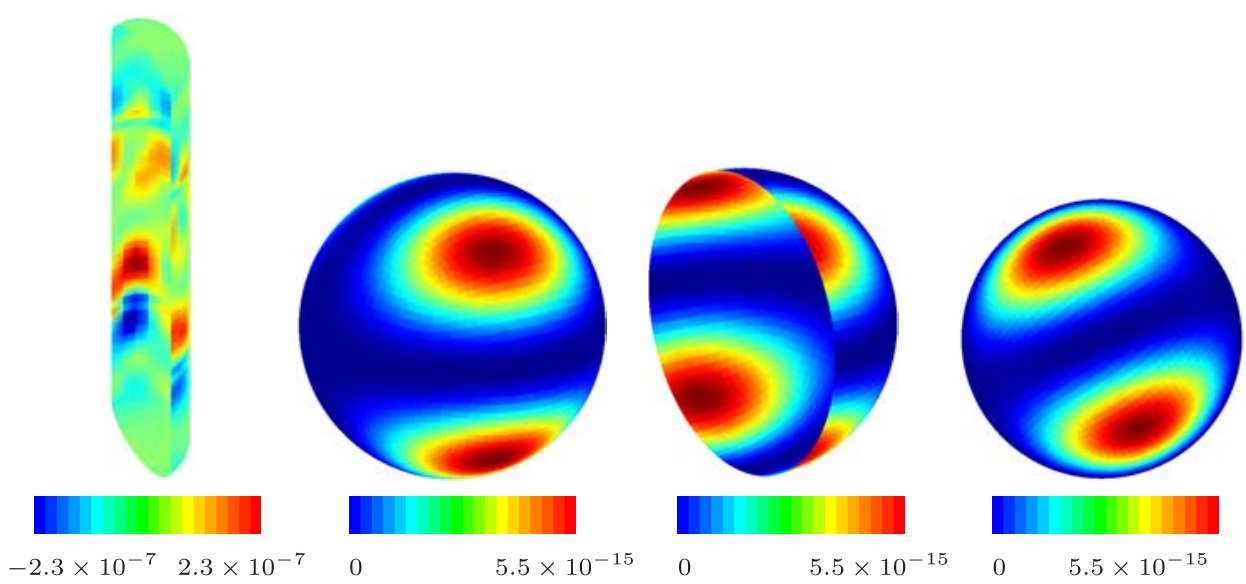

$\mathbf{I}_{\Gamma}^{\mathrm{NNI}}$

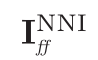

$\mathbf{I}_{f f}^{\mathrm{NNI}}$

$\mathbf{I}_{f f}^{\mathrm{NNI}}$
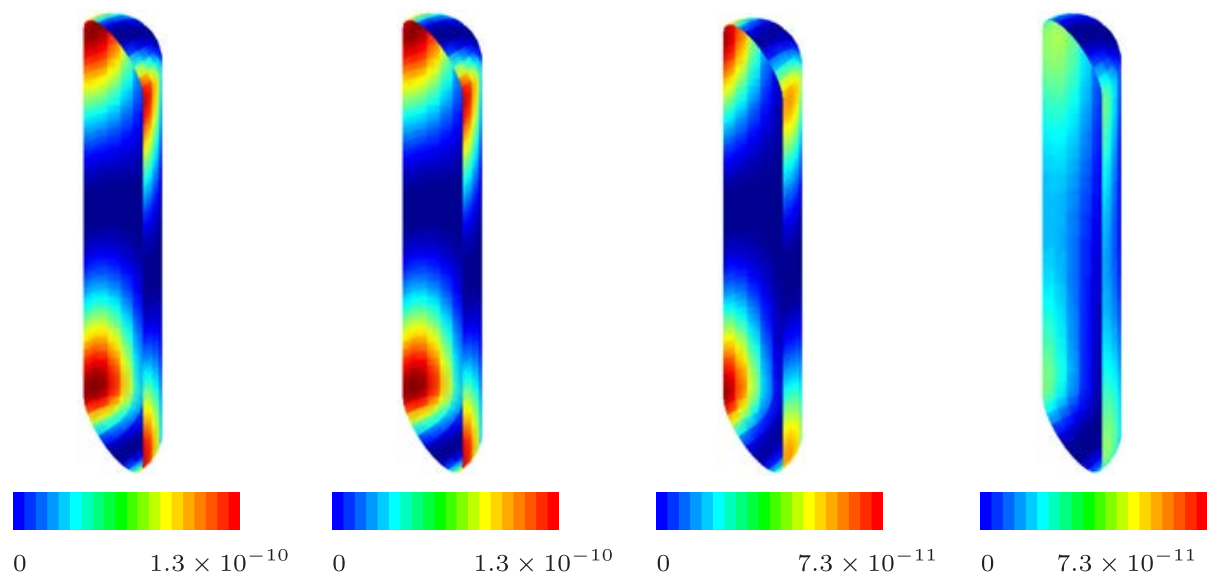

Fig. 14. Acoustic intensity at the structural surface $\mathbf{I}_{\Gamma}$, acoustic intensity at the far-field receiver surfaces $\mathbf{I}_{f f}$, NNI $\mathbf{I}_{\Gamma}^{\mathrm{NNI}}$, and back-calculated NNI $\mathbf{I}_{f f}^{\mathrm{NNI}}$, for the second bending complex mode of the cylindrical shell at $20 \mathrm{~Hz}$ (intensity in $\mathrm{W} / \mathrm{m}^{2}$ ). 
Case 4
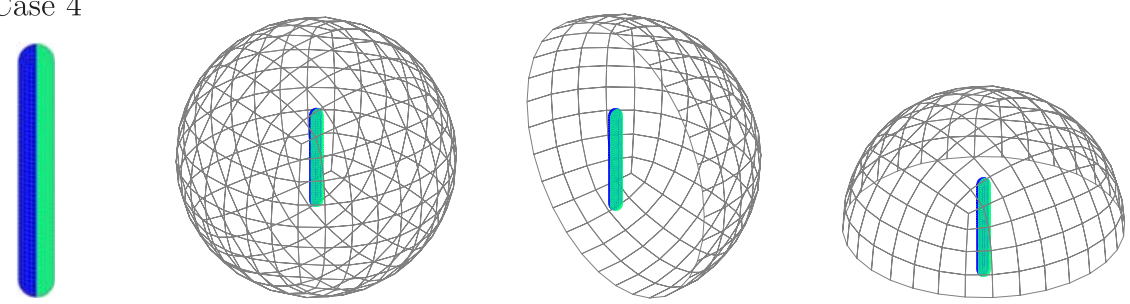

$\mathbf{I}_{\Gamma}$

$\mathbf{I}_{f f}$

$\mathbf{I}_{f f}$

$\mathbf{I}_{f f}$
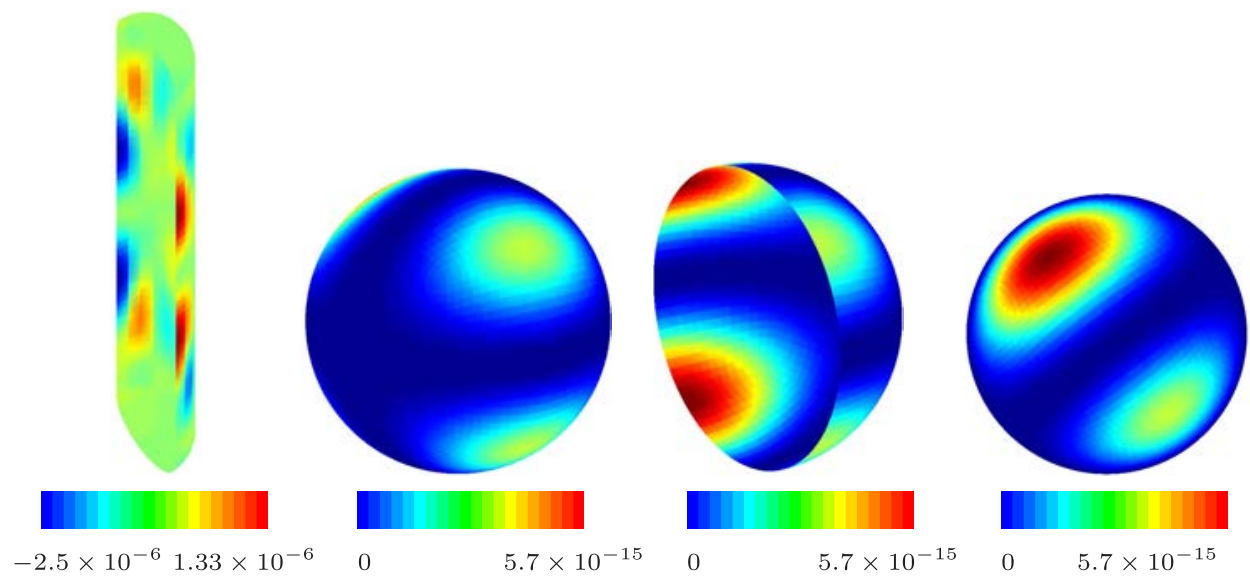

$-2.5 \times 10^{-6} 1.33 \times 10^{-6}$

0

$5.7 \times 10^{-15}$

0

$5.7 \times 10^{-15}$

$\mathbf{I}_{\Gamma}^{\mathrm{NNI}}$

$\mathbf{I}_{f f}^{\mathrm{NNI}}$

$\mathbf{I}_{f f}^{\mathrm{NNI}}$

$\mathbf{I}_{f f}^{\mathrm{NNI}}$
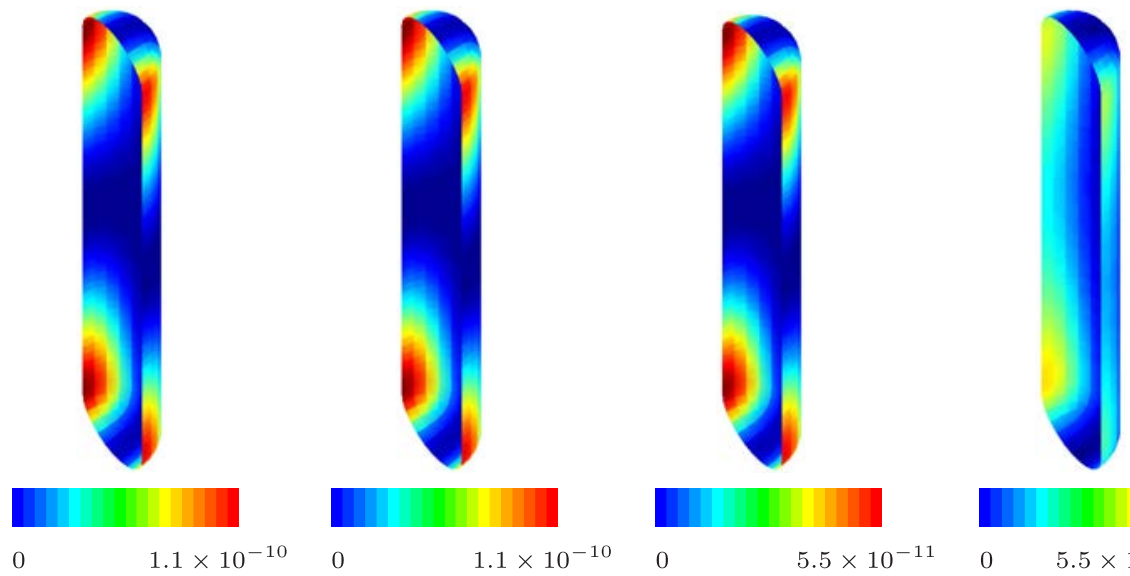

Fig. 15. Acoustic intensity at the structural surface $\mathbf{I}_{\Gamma}$, acoustic intensity at the far-field receiver surfaces $\mathbf{I}_{f f}$, NNI $\mathbf{I}_{\Gamma}^{\mathrm{NNI}}$, and back-calculated NNI $\mathbf{I}_{f f}^{\mathrm{NNI}}$, for the second bending complex mode of the cylindrical shell at $20 \mathrm{~Hz}$ (intensity in $\mathrm{W} / \mathrm{m}^{2}$ ). 
D. Liu et al.

Case 5
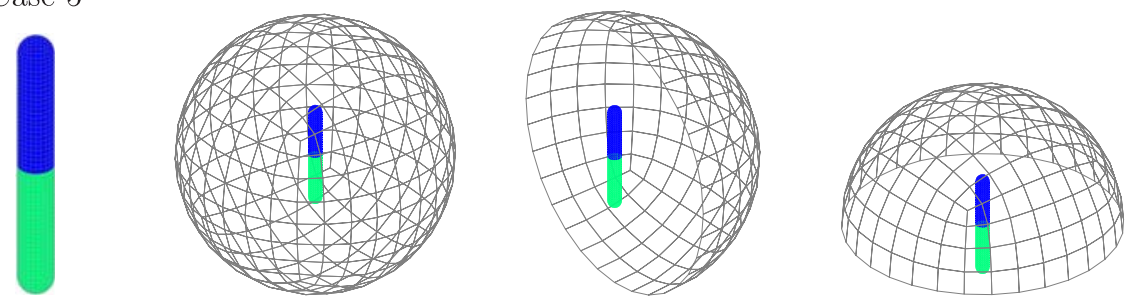

$\mathbf{I}_{\Gamma}$

$\mathbf{I}_{f f}$

$\mathbf{I}_{f f}$

$\mathbf{I}_{f f}$
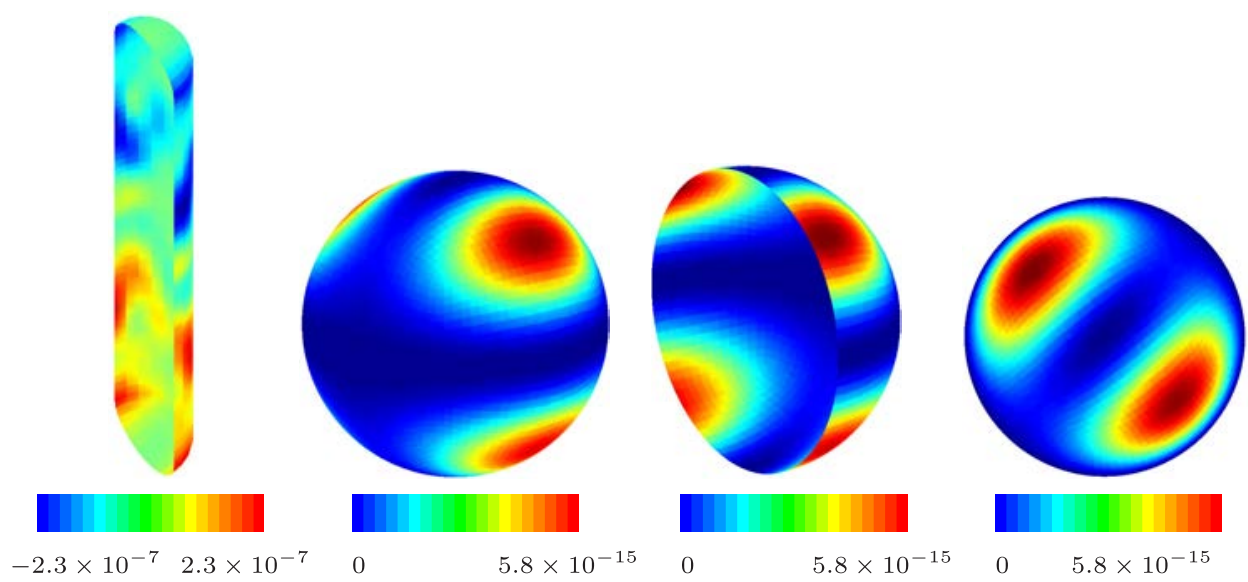

$\mathbf{I}_{\Gamma}^{\mathrm{NNI}}$

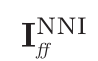

$\mathbf{I}_{f f}^{\mathrm{NNI}}$

$\mathbf{I}_{f f}^{\mathrm{NNI}}$
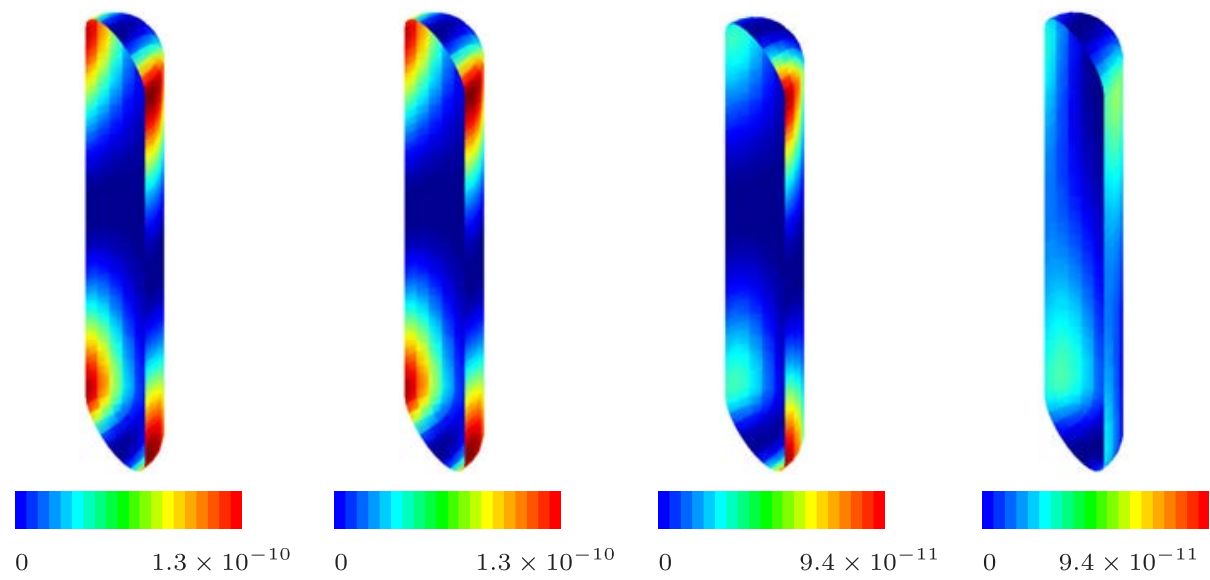

Fig. 16. Acoustic intensity at the structural surface $\mathbf{I}_{\Gamma}$, acoustic intensity at the far-field receiver surfaces $\mathbf{I}_{f f}$, NNI $\mathbf{I}_{\Gamma}^{\mathrm{NNI}}$, and back-calculated NNI $\mathbf{I}_{f f}^{\mathrm{NNI}}$, for the second bending complex mode of the cylindrical shell at $20 \mathrm{~Hz}$ (intensity in $\mathrm{W} / \mathrm{m}^{2}$ ). 
Case 6
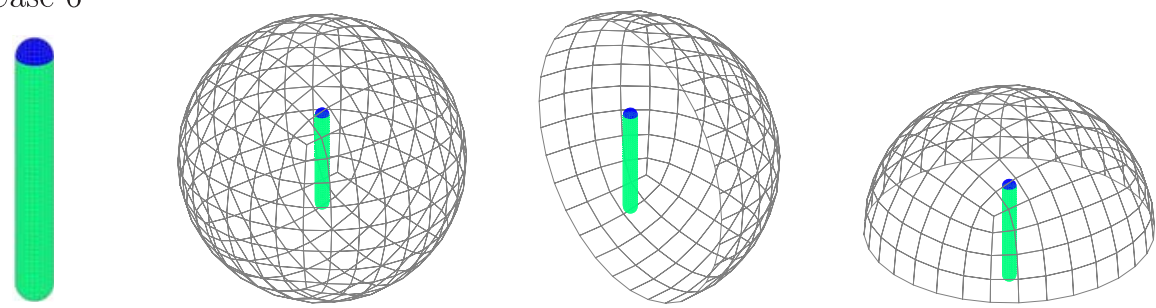

$\mathbf{I}_{\Gamma}$

$\mathbf{I}_{f f}$
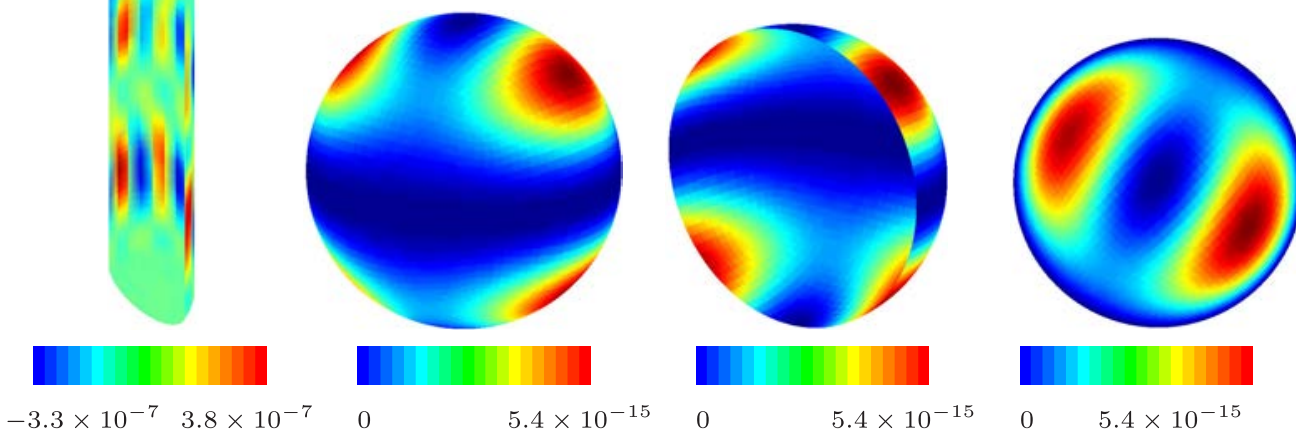

0

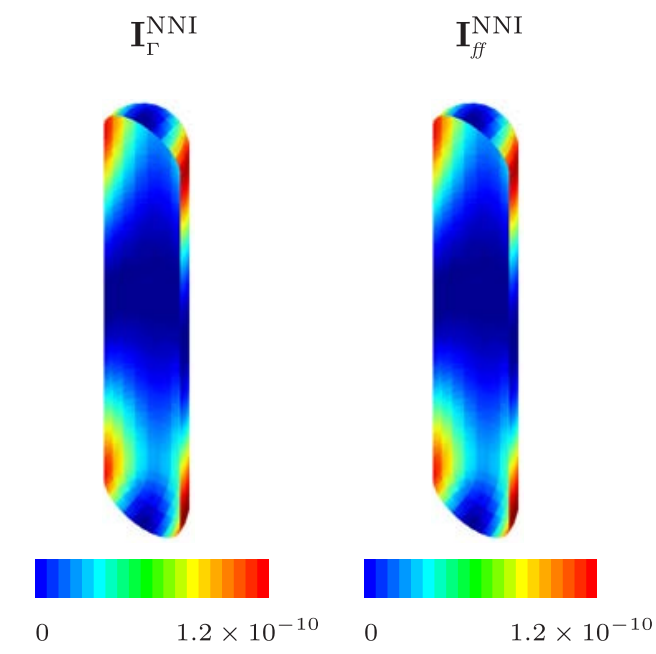

$\mathbf{I}_{f f}^{\mathrm{NNI}}$

$\mathbf{I}_{f f}^{\mathrm{NNI}}$
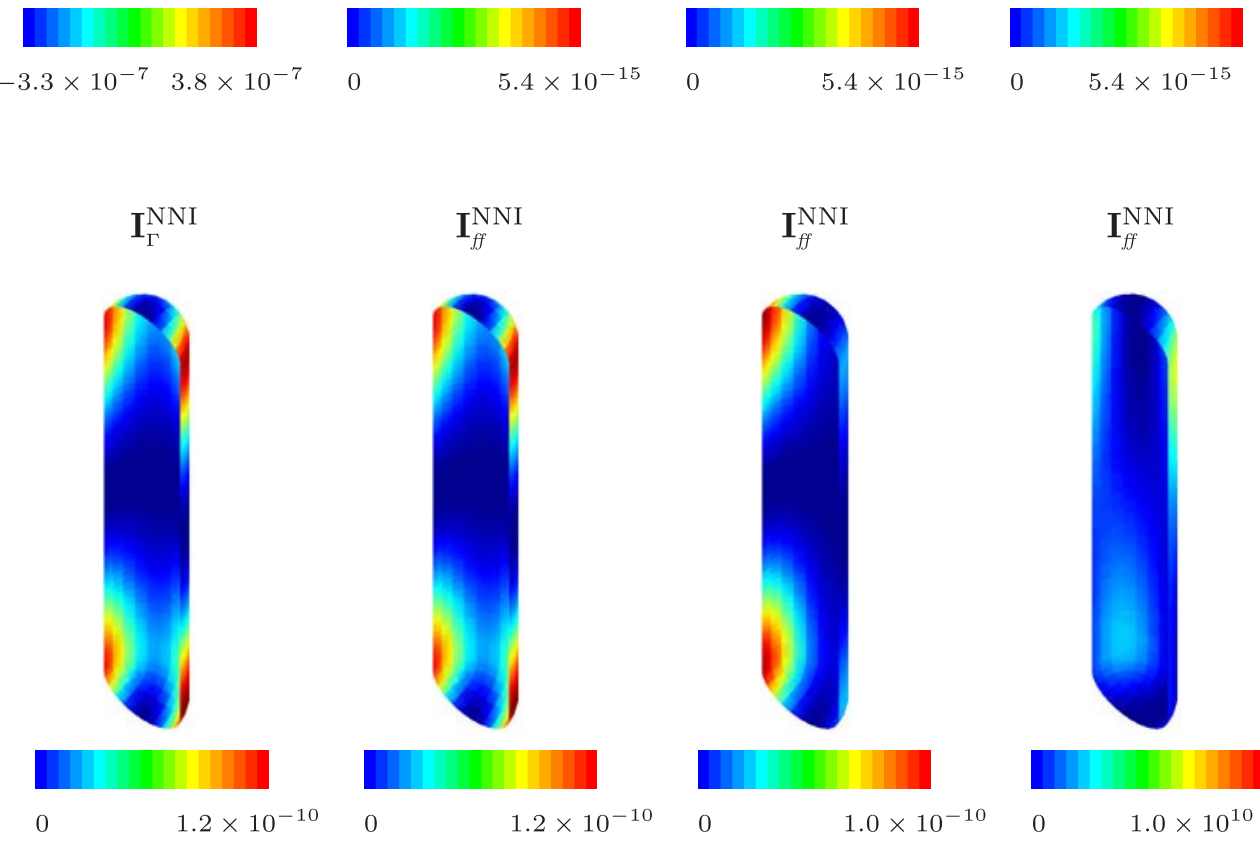

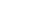

$\mathbf{I}_{f f}$

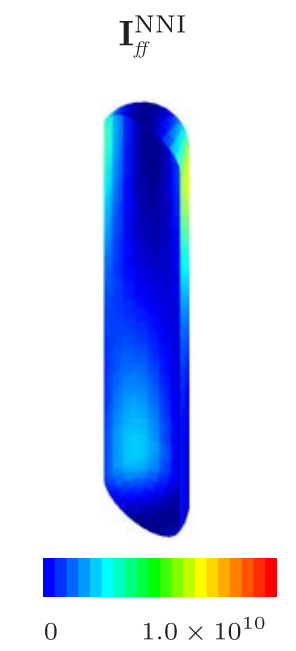

Fig. 17. Acoustic intensity at the structural surface $\mathbf{I}_{\Gamma}$, acoustic intensity at the far-field receiver surfaces $\mathbf{I}_{f f}$, NNI $\mathbf{I}_{\Gamma}^{\mathrm{NNI}}$, and back-calculated NNI $\mathbf{I}_{f f}^{\mathrm{NNI}}$, for the second bending complex mode of the cylindrical shell at $20 \mathrm{~Hz}$ (intensity in $\mathrm{W} / \mathrm{m}^{2}$ ). 


\section{Liu et al.}

\section{Conclusions}

In this work, the surface contributions to radiated sound power from elastic structures with inhomogeneous distributions of Rayleigh damping have been presented. To investigate the influence of inhomogeneous damping on the radiated sound power, several different damping configurations were applied to an odd-odd bending mode of a square plate and the second bending mode of a cylindrical shell. In the presence of inhomogeneous damping, the particle velocity was observed to include both standing and traveling waves, which generates complex vibration modes. Inspection of the real and imaginary parts of the particle velocity revealed traveling waves propagating to regions with higher damping. Active acoustic intensity on the structural surfaces comprised both positive and negative components, where the negative components always correspond to the higher damping regions for the cases of inhomogeneous damping. Regions with higher damping dissipate bending waves act as energy sinks, which in turn was shown to contribute more energy to the far-field acoustic intensity. Results for NNI and back-calculated NNI were shown to exhibit plate corner radiation and cylinder edge radiation due to near-field cancellation effects of acoustic energy in adjacent regions, at the low frequencies considered in this work. Doubly symmetric inhomogeneous damping distributions were observed to have very little influence on the far-field acoustic intensity and NNI compared to results with homogeneous damping for the simple examples considered here. The results presented here highlight that greatest NNI occurs at localized regions comprising both positive and negative acoustic intensity on the surface of the structure. Due to its ability to avoid cancellation effects between positive and negative values of acoustic intensity from adjacent areas, NNI can assist in the application of inhomogeneous damping for noise control.

\section{References}

1. E. G. Williams, H. D. Dardy and K. B. Washburn, Generalized nearfield acoustical holography for cylindrical geometry: Theory and experiment, J. Acoust. Soc. Am. 81(2) (1987) 389-407.

2. E. G. Williams, Supersonic acoustic intensity, J. Acoust. Soc. Am. 97(1) (1995) 121-127.

3. E. G. Williams, Supersonic acoustic intensity on planar sources, J. Acoust. Soc. Am. 104(5) (1998) 2845-2850.

4. E. Fernandez-Grande, F. Jacobsen and Q. Leclère, Direct formulation of the supersonic acoustic intensity in space domain, J. Acoust. Soc. Am. 131(1) (2012) 186-193.

5. E. Fernandez-Grande and F. Jacobsen, Conservation of power of the supersonic acoustic intensity, J. Acoust. Soc. Am. 136(2) (2014) 461-465.

6. M. B. S. Magalhães and R. A. Tenenbaum, Supersonic acoustic intensity for arbitrarily shaped sources, Acta Acust. United Acust. 92 (2006) 189-201.

7. C. A. Corrêa Junior and R. A. Tenenbaum, Useful intensity: A technique to identify radiating regions on arbitrarily shaped surfaces, J. Sound Vib. 332(6) (2013) 1567-1584.

8. N. P. Valdivia, E. G. Williams and P. C. Herdic, Equivalent sources method for supersonic intensity of arbitrarily shaped geometries, J. Sound Vib. 347 (2015) 46-62.

9. S. Marburg, E. Lösche, H. Peters and N. Kessissoglou, Surface contributions to radiated sound power, J. Acoust. Soc. Am. 133(6) (2013) 3700-3705.

10. E. G. Williams, Convolution formulations for non-negative intensity, J. Acoust. Soc. Am. 134(2) (2013) 1055-1066. 
11. D. Liu, H. Peters, S. Marburg and N. Kessissoglou, Supersonic intensity and non-negative intensity for prediction of radiated sound, J. Acoust. Soc. Am. 139(5) (2016) 2797-2806.

12. D. Liu, Z. Havránek, S. Marburg, H. Peters and N. Kessissoglou, Non-negative intensity and back-calculated non-negative intensity for analysis of directional structure-borne sound, $J$. Acoust. Soc. Am. 142(1) (2017) 117-123.

13. D. R. Wilkes, H. Peters, P. Croaker, S. Marburg, A. J. Duncan and N. Kessissoglou, Non-negative intensity for coupled fluid-structure interaction problems using the fast multipole method, $J$. Acoust. Soc. Am. 141(6) (2017) 4278-4288.

14. D. Liu, H. Peters, S. Marburg and N. Kessissoglou, Surface contributions to scattered sound power using non-negative intensity, J. Acoust. Soc. Am. 140(2) (2016) 1206-1217.

15. G. F. Lang, Demystifying complex modes, Sound Vib. 23(1) (1989) 36-40.

16. S. Marburg, Normal modes in external acoustics. Part III: Sound power evaluation based on superposition of frequency-independent modes, Acta Acust. United Acust. 92(2) (2006) 296-311.

17. O. Unruh, M. Sinapius and H. P. Monner, Sound radiation properties of complex modes in rectangular plates: A numerical study, Acta Acust. United Acust. 101(1) (2015) 62-72.

18. O. Unruh, Parametric study of sound radiation properties of complex vibration patterns in rectangular plates using an analytical model, Acta Acust. United Acust. 101(4) (2015) 701-712.

19. O. Unruh, Influence of inhomogeneous damping distribution on sound radiation properties of complex vibration modes in rectangular plates, J. Sound Vib. 377 (2016) 169-184.

20. S. Marburg and B. Nolte, Computational Acoustics of Noise Propagation in Fluids - Finite and Boundary Element Methods (Springer-Verlag, Berlin, Heidelberg, 2008), pp. 1-34.

21. H. Peters, N. Kessissoglou and S. Marburg, Enforcing reciprocity in numerical analysis of acoustic radiation modes and sound power evaluation, J. Comput. Acoust. 20(3) (2012) 1250005.

22. H. Peters, S. Marburg and N. Kessissoglou, Structural-acoustic coupling on non-conforming meshes with quadratic shape functions, Int. J. Numer. Methods Eng. 91(1) (2012) 27-38.

23. S. Marburg and T. W. Wu, Computational Acoustics of Noise Propagation in Fluids — Finite and Boundary Element Methods (Springer-Verlag, Berlin, Heidelberg, 2008), pp. 411-424.

24. C. W. de Silva, Vibration: Fundamentals and Practice (CRC Press, 2000).

25. ANSYS, Theory Reference for the Mechanical APDL and Mechanical Applications, ANSYS Inc., Canonsburg, Pennsylvania, USA, release 12.1 (2009).

26. S. Marburg, Numerical damping in the acoustic boundary element method, Acta Acust. United Acust. 102(3) (2016) 415-418.

27. S. Marburg, The Burton and Miller method: Unlocking another mystery of its coupling parameter, J. Comput. Acoust. 24(1) (2016) 1550016.

28. G. Maidanik, Response of ribbed panels to reverberant acoustic fields, J. Acoust. Soc. Am. 34(6) (1962) 809-826. 